ISSN: 0514-7336

DOI: http://dx.doi.org/10.14201/zephyrus2015751739

\title{
LA 'GALERÍA DE LOS BISONTES': UN NUEVO SECTOR DECORADO EN LA CUEVA DE ALKERDI (URDAZUBI/URDAX, NAVARRA)
}

\section{The Bisons Gallery: a new decorated sector in Alkerdi cave (Urdazubi/Urdax, Navarra)}

\author{
Diego Garate Maidagan* y Olivia Rivero Vilák* \\ * Arkeologi Museoa-Museo Arqueológico de Vizcaya. C/ Calzadas de Mallona, 2. 48006 Bilbao. Correo-e: \\ garatemaidagandiego@gmail.com \\ ** Instituto Internacional de Investigaciones Prehistóricas de Cantabria. Universidad de Cantabria. Avda. de los \\ Castros, s/n. 39005 Santander. Correo-e: oliviariver@hotmail.com
}

Recepción: 15/02/2015; Revisión: 6/03/2015; Aceptación: 5/04/2015

BIBLID [0514-7336 (2015) LXXV, enero-junio; 17-39]

\begin{abstract}
Resumen: Alkerdi es la única cueva con grabados parietales paleolíticos conocida en la Comunidad Foral de Navarra. Situada en el piedemonte pirenaico, su descubrimiento fue protagonizado por el espeleólogo francés N. Casteret en 1930, quien lo publicó, de manera muy breve, poco después (1933). El estudio detallado de los grabados no tendrá lugar hasta varias décadas más tarde, de la mano de I. Barandiarán (1974), quien, en los últimos ańos, ha excavado el yacimiento arqueológico de la cavidad (2009). Durante 2014 los firmantes han desarrollado un nuevo estudio de su arte parietal ante el hallazgo de una nueva galería decorada en la que se concentra una veintena de figuras grabadas, mayoritariamente representaciones de bisontes, que multiplican exponencialmente el conjunto anteriormente conocido. Las convenciones y características formales de las figuras responden a las propias del Magdaleniense medio, especialmente vinculadas a los conjuntos de la vertiente pirenaica. El giro producido en el conocimiento del arte parietal de la cavidad obliga a reevaluar su papel en esta área bisagra de los Pirineos occidentales.

Palabras clave: Arte rupestre; grabado; Paleolítico superior; Cantábrico; Pirenaico.
\end{abstract}

AвsтRACT: Alkerdi is the only cave with Paleolithic rock art engravings known in the Regional Community of Navarre. Located in Pyrenean foothills, its discovery was carried out by the French speleologist N. Casteret in 1930, who published it briefly shortly after (1933). The detailed study of the engravings will not take place until several decades after, by I. Barandiarán (1974) who, in the last years, has excavated the archaeological deposit of the cavity (2009). During 2014 the signers have developed a new study of the parietal art because of the finding of a new decorated gallery in which there are concentrated twenty engraved figures, mainly representations of bison, which exponentially multiply the previously known set. The conventions and formal characteristics of the figures respond to those ones of the Middle Magdalenian, and especially tie to the sets of the Pyrenean region. This new turn in the knowledge of the parietal art of the cavity forces a re-evaluation of the study in this area hinged in the western Pyrenees.

Key words: Rock art; engravings; Upper Palaeolithic; Cantabrian region; Pyrenean.

(C) Universidad de Salamanca

Zephyrus, LXXV, enero-junio 2015, 17-39 


\section{Los estudios previos en la cavidad ${ }^{1}$}

La cueva de Alkerdi (Urdazubi/Urdax) se localiza en el margen derecho de la cuenca alta del río La Nivelle/Ugarana, en la cabeza de una serie de arroyos que lo drenan. Por lo tanto, el yacimiento se sitúa geográficamente en la vertiente norte de la cordillera pirenaica (Fig. 1).
La boca, orientada al s, se abre encajada en el farallón calizo de Zelaieta y frente a las faldas del monte Arleun. En ese mismo emplazamiento, a escasa distancia, se encuentran Alkerdi 2, con un depósito funerario de la prehistoria reciente (Barandiarán et al., 2010), y el abrigo de Berroberria, cuyo yacimiento arqueológico ha sido excavado sucesivamente por el marqués de Loriana en 1939 (1940),

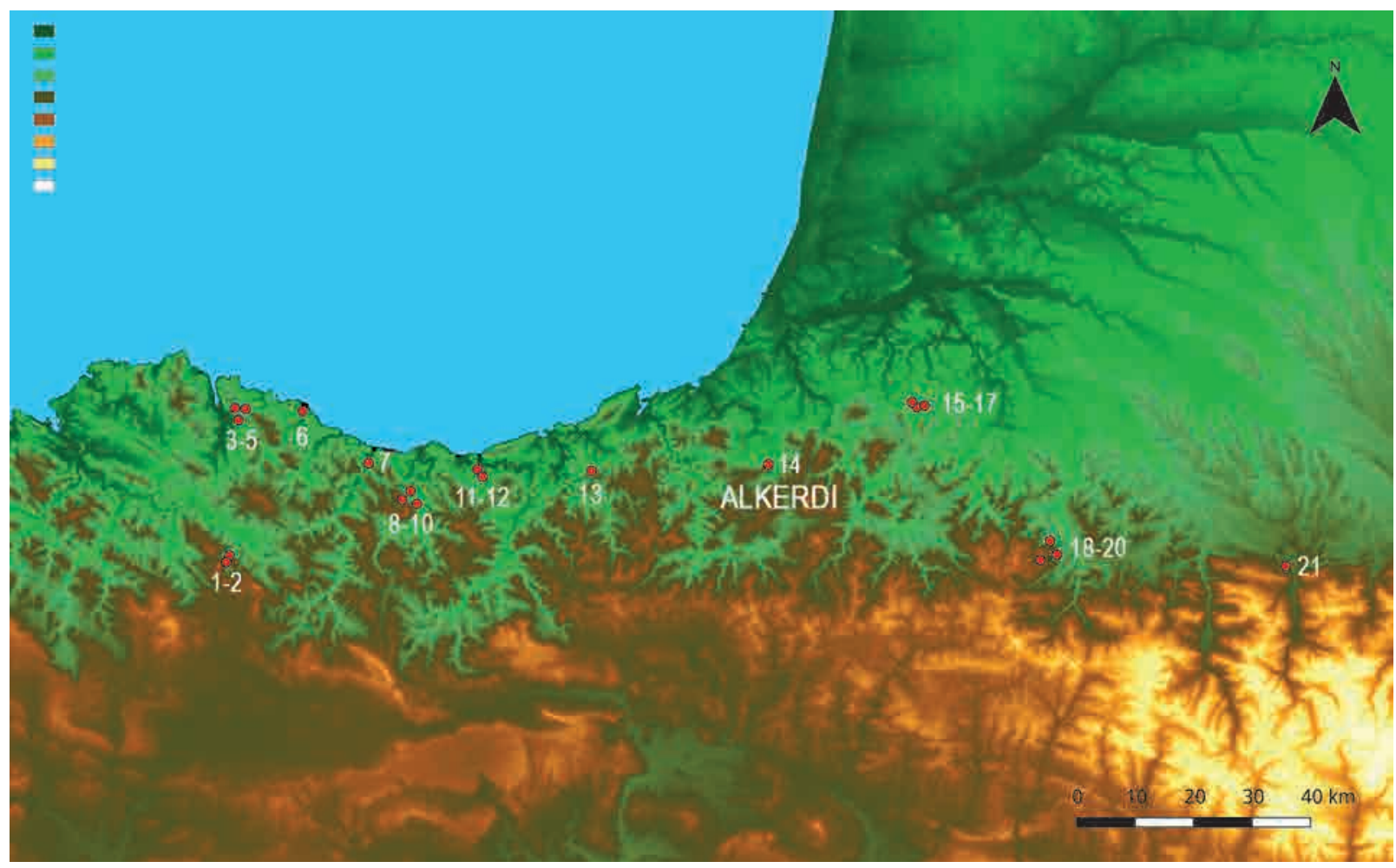

Fig. 1. Localización de la cueva decorada de Alkerdi (Urdazubi/Urdax, Navarra) en los Pirineos occidentales: 1) Atxuri; 2) Askondo; 3) Morgota; 4) Antoliña; 5) Santimamiñe; 6) Lumentxa; 7) Praile Aitz; 8) Astigarraga; 9) Danbolinzulo; 10) Ekain; 11) Altxerri A; 12) Altxerri B; 13) Aitzbitarte IV; 14) Alkerdi; 15) Isturitz; 16) Oxocelhaya; 17) Erberua; 18) Sasiziloaga; 19) Sinhikole; 20) Etxeberri; 21) Sainte-Colome.

${ }^{1}$ Los autores del artículo agradecen al Servicio de Patrimonio Histórico, Dpto. de Cultura, Turismo y Relaciones Institucionales del Gobierno de Navarra (arqueólogos J. Sesma Sesma y J. García Gazolaz) por autorizar y subvencionar la actuación. Al Ayto. de Urdazubi/Urdax (alcalde S. Villares) por facilitar nuestro trabajo y valorar el interés patrimonial del mismo. También quieren expresar su agradecimiento a $\mathrm{V}$. Bayarri y J. Herrera, de Gimgeomatics, por su dedicación y, especialmente, a G. Hualde, A. Ollo y J. Sarratea del centro "Cuevas de Urdax/Urdazubi lezeak" por su colaboración constante y su calurosa acogida.
J. Maluquer de Motes entre 1959 y 1964 (1965) y finalmente I. Barandiarán y A. Cava entre 1977 y 1994 (Barandiarán et al., 2010), evidenciando una importante secuencia para los momentos finales del Paleolítico superior. Un poco más alejadas, al otro lado del farallón, se encuentran las cuevas de Zelaieta, una de las cuales presentaba vestigios arqueológicos de la Prehistoria reciente (Casteret, 1933) y que actualmente se encuentran gravemente afectadas y/o destruidas por la cantera contigua. 
La entrada a la cavidad queda protegida por el frente calizo. De forma arqueada, presenta unas dimensiones aproximadas de $6 \mathrm{~m}$ de ancho y 3,5 de altura máxima. Se accede así a un vestíbulo de techos muy bajos, apenas 1,5 m en muchos puntos, pero de una amplitud considerable con 8 metros de anchura y hasta 10 metros de profundidad. A la izquierda parte una galería estrecha pero de techos altos que finaliza en un recodo, donde se concentra el arte parietal conocido y publicado hasta la fecha. Hacia la derecha, una serie de formaciones estalagmíticas dificulta el paso a una segunda sala de similares características a la del vestíbulo, con techos todavía más bajos y unas dimensiones aproximadas de $14 \times 6 \mathrm{~m}$. Al fondo de este segundo espacio, parte de la pared izquierda una estrecha galería cuyas dimensiones decrecen progresivamente hasta convertirse en una gatera de difícil tránsito en su tramo

final, donde se vuelve a unir con la mencionada sala. Es en este lugar donde se concentran los grabados recientemente descubiertos. Por último, también desde la parte derecha del vestíbulo, pasando un estrecho conducto y una gatera muy reducida, se asciende por una sima que conecta la cueva con el exterior.

El arte parietal paleolítico de la cavidad fue descubierto por N. Casteret en 1930 durante una visita a varias cuevas en los municipios de Zugarramurdi y Urdazubi/Urdax, en el extremo norte de la Comunidad Foral de Navarra. Los resultados de dicho hallazgo son descritos de manera muy breve e incluso confusa en una nota publicada poco después (1933). En ella se describe la cavidad a grandes rasgos y, de hecho, se menciona ya la existencia de ..."une mauvaise tête de bovidé"... en la gatera donde se concentran los nuevos hallazgos que presentamos, espacio que también se indica con una ' $\mathrm{x}$ ' y un ' 4 ' en el plano aportado. Esa cabeza de bisonte se incluye en la fig. 3 (n. ${ }^{\circ}$ ) de su publicación pero, inexplicablemente, ha pasado desapercibida hasta la actualidad (Fig. 2).
Lo mismo sucede con la crinera de caballo (n. ${ }^{\circ} 5$ de la misma fig. 3) que es dibujada por N. Casteret, pero sin mencionar en el texto su ubicación en la cueva y que hemos reconocido también en la galería decorada que exponemos más adelante. En el extremo opuesto de la cavidad es donde sitúa el conjunto principal de representaciones grabadas: un ciervo, una cabeza de Bos primigenius, un tren trasero de bóvido y otro de caballo. El descubridor considera que ..."ils sont assez frustes et ne rappellent en rien les admirables fresques de certaines grottes”... aunque también señala que la corrosión del soporte los ha degradado, resultando así poco vistosos.

En 1935, J. C. Baroja realiza calcos de los grabados por encargo de J. M. Barandiarán (Barandiarán Irizar, 1989), quien ese mismo año señala la existencia del yacimiento arqueológico de la cavidad (Barandiarán, 1946) ${ }^{2}$.

${ }^{2}$ No hemos podido precisar la fecha exacta debido a la pérdida del diario personal de J. M. Barandiarán correspondiente al año 1935 (Barandiarán, 2005). 


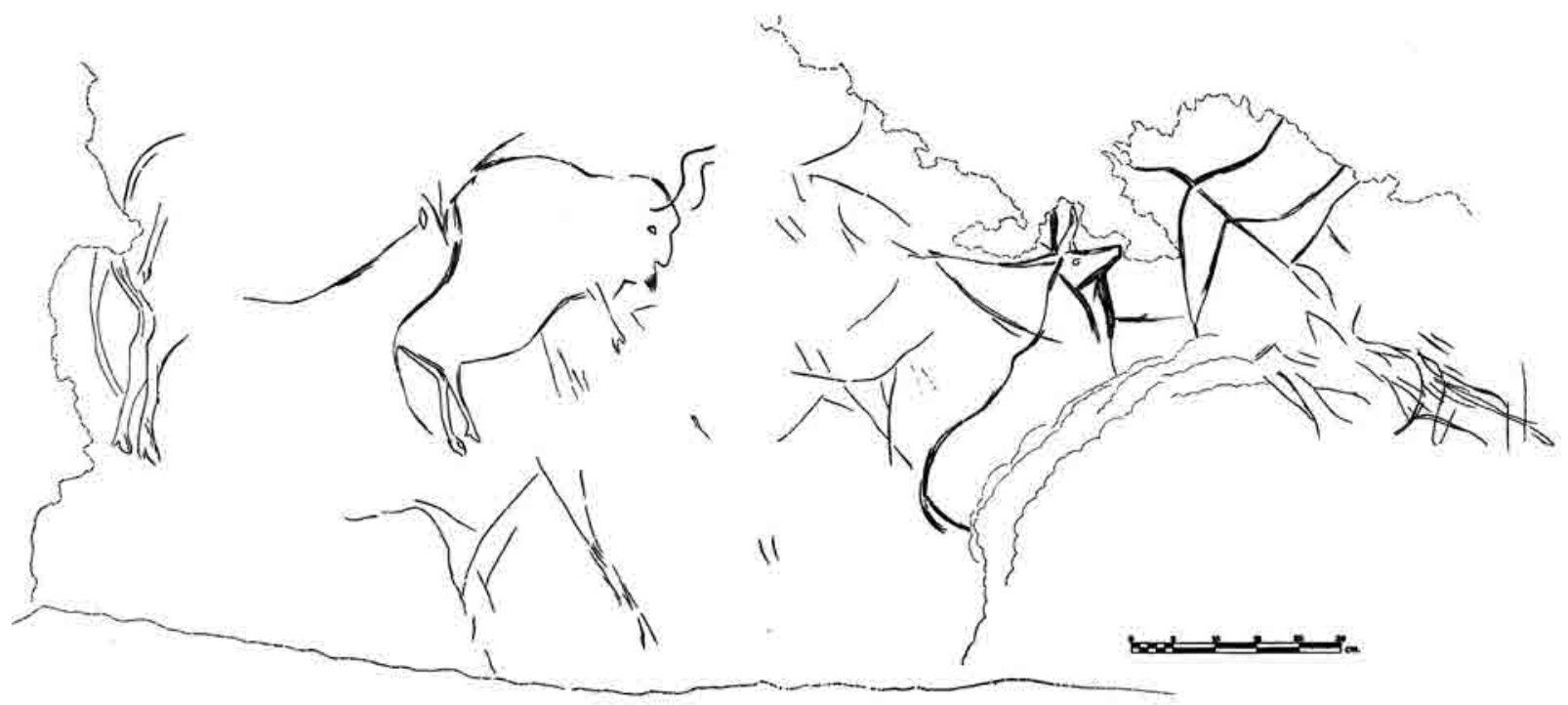

FIG. 3. Calco directo del conjunto 1 (según Barandiarán, 1974).

En 1939 la cueva será de nuevo visitada por N. Casteret junto al marqués de Loriana, quien se limita a realizar nuevos calcos de algunas de las figuras del sector izquierdo y a recoger algunos materiales arqueológicos (1940). Durante los siguientes ańos el interés arqueológico se centrará en el abrigo contiguo de Berroberria, como ya hemos comentado, hasta la revisión efectuada por I. Barandiarán en 1973 de los grabados parietales de Alkerdi. El estudio será publicado de manera inmediata (Barandiarán, 1974), ofreciendo una descripción exhaustiva de las figuras incluyendo su posicionamiento en una nueva topografía más detallada aunque limitada al sector izquierdo de la cueva, un estudio tecnológico a través de moldes tomados directamente de los grabados y calcos de todos los motivos incluyendo la comparativa con las versiones anteriores (Fig. 3). Al mismo tiempo, se añaden dos nuevas figuras localizadas en el vestíbulo de la cavidad, pero no se localiza el bisonte señalado por N. Casteret en el sector derecho de la misma ni la crinera de caballo. Con posterioridad las intervenciones arqueológicas en el yacimiento se han ceñido a su depósito arqueológico excavado por I. Barandiarán y A. Cava durante los años 1988, 1993 y 1994. La extensión abierta comprendía un espacio de $11 \mathrm{~m}^{2}$ en los que se identificó un nivel de ocupación, poco intensa, atribuido al Gravetiense en función de su cultura material y de una datación radiocarbónica sobre hueso en 26470 $\pm 530 / 490$ в - GrN20322- (Cava et al., 2009).

El 9 de marzo de 2014, en el marco de las visitas guiadas organizadas desde el Ayto. de Urdazubi/Urdax con motivo de la celebración del Día Internacional de las Cuevas, los arriba firmantes accedieron a la cavidad, identificando los grabados en una galería lateral. Dicha galería aparecía señalada con una marca ' $\mathrm{x}$ ' en la publicación original de N. Casteret y respondía al espacio donde señalaba la existencia de "une mauvaise tête de bovidé" (1933). Durante la visita se localizó dicha figura en la galería, así como otras figuras grabadas de bisontes. Considerando la especial relevancia del hallazgo, se procedió a dar cuenta a las administraciones competentes y, seguidamente, se solicitó una autorización de estudio de arte rupestre ${ }^{3}$. Durante el segundo semestre de 2014 se desarrolló la intervención siguiendo las pautas señaladas a continuación y que permitió localizar una línea cérvico-dorsal de animal en el pasillo de acceso - B- a la salita de la izquierda donde se encontraban los grabados publicados por N. Casteret (1933) e I. Barandiarán (1974) y, por otro

3 El lunes 5 de mayo se comunicó al Dpto. de Cultura y Turismo del Gobierno de Navarra. El día 13 de junio la solicitud de autorización (180/2014) fue resuelta favorablemente. 

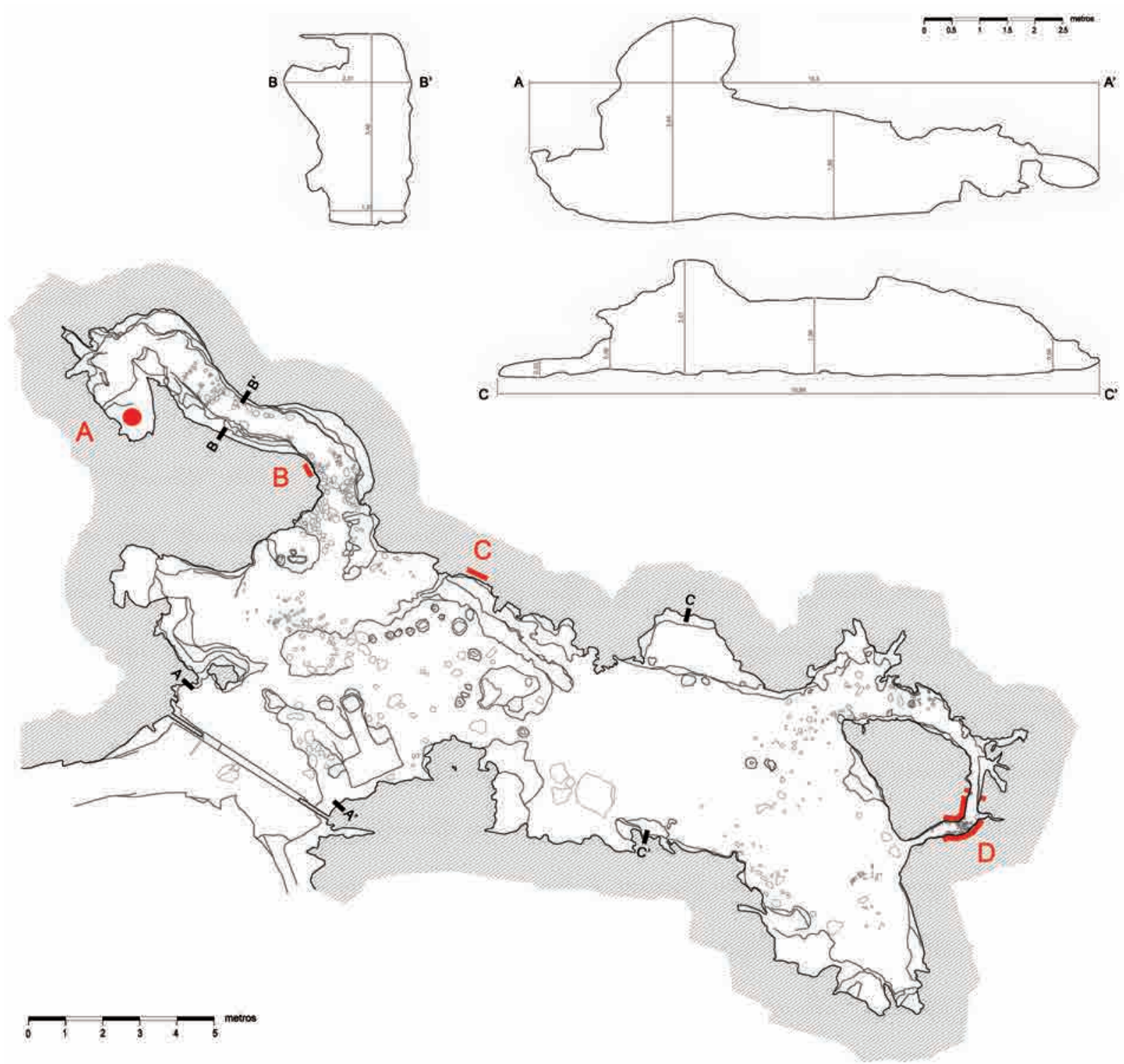

Fig. 4. Planimetría precisa de la cueva con indicación de los sectores decorados (A, B, C y D).

lado, veinte motivos, uno de ellos pintado y el resto grabados, en la galería lateral $-\mathrm{D}-$ de la sala situada a derecha, donde N. Casteret (1933) ya señaló la presencia de grabados (Fig. 4).

\section{Metodología del estudio}

El nuevo sector decorado descubierto en la cueva de Alkerdi presenta una serie de características que precisan de una metodología de trabajo específica y adaptada a las condiciones del sitio para la documentación de los motivos parietales bajo los planteamientos metodológicos actuales (Fritz y Tosello, 2007). Por un lado, se trata de representaciones trazadas mediante grabado extremadamente fino, de lectura muy compleja, así como difíciles de captar fotográficamente. Por otro lado, las reducidas dimensiones del espacio, que apenas permiten albergar a una persona en posturas forzadas, condicionan enormemente todas las labores de documentación del arte parietal y su propia observación. 


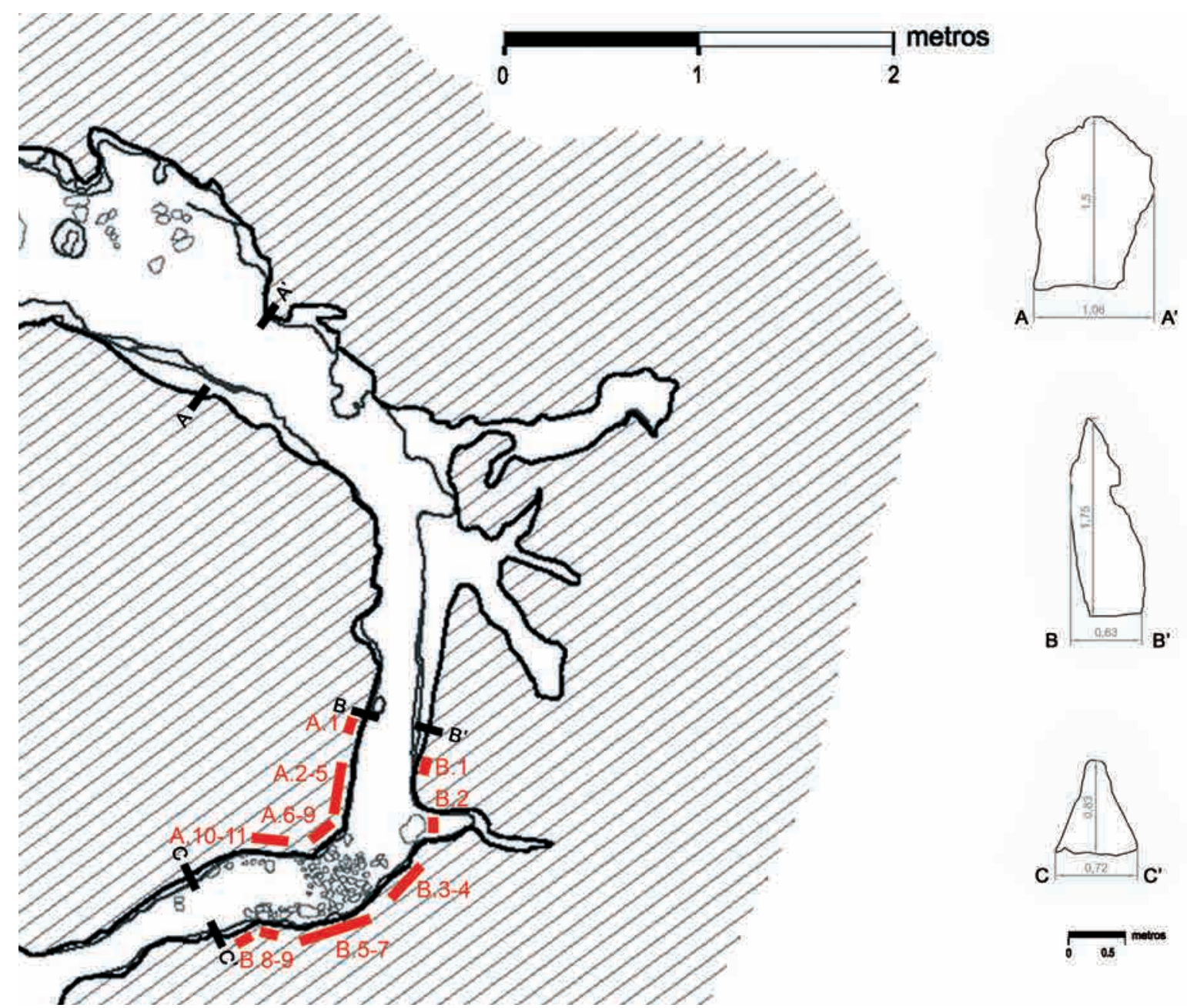

FIG. 5. Detalle de la planimetría con la posición de los motivos descubiertos en la galería lateral (sector D).

La lectura de los grabados se abordó contando con diversos sistemas de iluminación como luces frontales de Led spot de $360 \mathrm{Lm}$ y Led de luz ambiental de $550 \mathrm{Lm}$, haciendo un total de $910 \mathrm{Lm}$ -Scurion ${ }^{\oplus} 900$ headlamp, Petzl ${ }^{\oplus}$ Duo Led 14 Accu headlamp, Led Lenser ${ }^{\oplus}$ M7R-, y focos transportables de batería autónoma con 6 Leds de alta intensidad de 5 vatios, valor lumínico comprobado 2000 -equivalente a una potencia lumínica total de 500 vatios- y régimen de color aproximado de $6000 \mathrm{k}$ -Peli 9430 Rals-. En todos los casos se trata de iluminación fría que no altera las condiciones ambientales de la cavidad. Asimismo, se recurrió a un microscopio portátil para la lectura y documentación de superposiciones y otros detalles de los grabados -Dino-lite ${ }^{\circledast}$ AD-7013mzT 30x-200x-.
Para la documentación gráfica contamos con una cámara digital Nikon ${ }^{\odot}$ D-7100, incorporando dos flashes Nikon ${ }^{\odot}$ sB-900 activados mediante Nikon ${ }^{\odot}$ wireless commander su-800 para crear haces de luz rasante, con un objetivo Nikon Nikkor ${ }^{\odot} 18-105 \mathrm{~mm}$ f3.5-5.6 AF-s DX para captar las figuras completas mediante el montaje de mosaicos, con un objetivo macrofotográfico Nikon Nikkor ${ }^{\odot} 40 \mathrm{~mm}$ f2.8 AF-S Micro para los detalles específicos y con otro objetivo gran angular Nikon Nikkor ${ }^{\odot}$ 10-24mm f3.5-4.5 AF-S DX para las tomas más generales.

La restitución gráfica de los grabados fue procesada mediante tratamiento digital de las imágenes y mediante ortofotografías obtenidas a través del programa Agisoft Photoscan ${ }^{\odot}$. Los calcos se han realizado manualmente in situ sobre fotografía a tamaño 
real y, posteriormente, infografía - paquetes informáticos de Adobe Photoshop ${ }^{\mathcal{O}}$ - para la digitalización de los calcos y generación de un soporte de fondo. Evidentemente, gracias al sistema diseñado, en ningún caso se tocaron las paredes y/o los motivos gráficos.

Por otro lado, el escaneo mediante Laser Scanner Terrestre 3D Faro ${ }^{\circledR}$ Photon 120 de toda la cavidad, excepto las gateras impracticables, permitió generar un plano y secciones nuevos de la cueva, así como un modelo 3D de la misma, que en el caso de la 'Galería de los Bisontes' fue texturizado mediante fotografías y colocación de los calcos de los grabados sobre las mismas.

\section{La 'Galería de los Bisontes': un nuevo sector decorado}

Como hemos mencionado con anterioridad, el nuevo sector decorado de la cueva de Alkerdi se localiza en el extremo opuesto con respecto al grueso del conjunto descubierto por N. Casteret en 1930. Para acceder es preciso dejar atrás el vestíbulo de la cavidad y adentrarse a la derecha en una sala amplia de techos bajos y suelo liso arcilloso. De la pared izquierda parte una galería de pequeñas dimensiones (Fig. 5), con unos $1,5 \times 1,5 \mathrm{~m}$ en su tramo inicial, que se estrecha de manera progresiva y que tras $3 \mathrm{~m}$ de desarrollo gira de orientación a la derecha convirtiéndose ya en un tubo de menos de $0,5 \mathrm{~m}$ de anchura y 1,5 de altura. Aquí, en unos $2 \mathrm{~m}$ de desarrollo, se localizan las primeras evidencias gráficas, donde el conducto vuelve a girar a la derecha hasta unirse de nuevo a la pared izquierda de la sala. En esta parte final de 1,5 m siguen disminuyendo las dimensiones hasta el punto

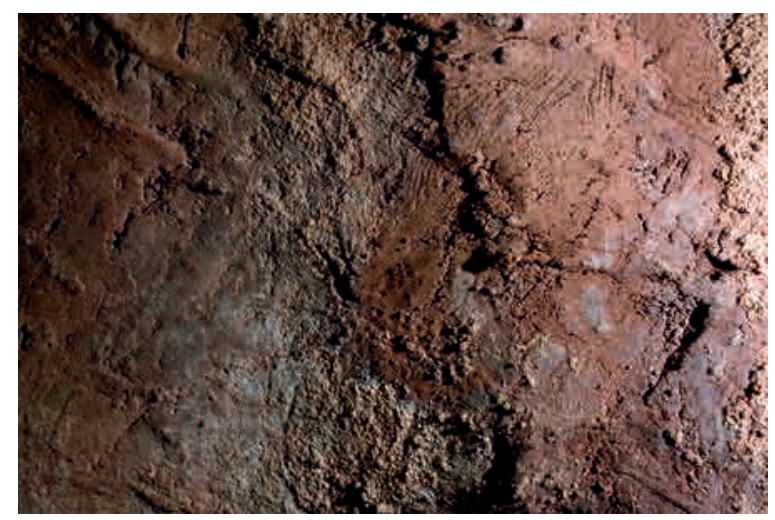

de impedir completamente el paso de vuelta a la sala, excepto a personas de pequeña talla. La mayoría de los grabados se concentran en el recodo que une los dos tramos finales, donde el observador se tiene que situar recostado y donde difícilmente hay aforo para más de una persona simultáneamente.

\subsection{Pared derecha (A)}

La pared derecha de la gatera presenta una sección rectilínea en el primer tramo de unos $2 \mathrm{~m}$ y en forma de tubo a partir del recodo, donde las dimensiones del conducto son ya mínimas. En este primer tramo se observan numerosas reconstrucciones espeleotémicas y la circulación hídrica sobre la superficie de la roca es constante. El segundo es más seco y el soporte calizo está menos cubierto.

- Caballo (A.1) (Fig. 6). Dimensiones: 28 × 8 $\mathrm{cm}$; altura al suelo: $83 \mathrm{~cm}$

Caballo incompleto orientado a la derecha y situado aislado al inicio del sector decorado de la galería. Grabado mediante incisiones poco profundas yuxtapuestas, presenta oreja, la crinera formada por líneas curvas paralelas y la línea cervicodorsal, así como el arranque del maxilar. Un trazo paralelo a la línea del lomo podría pertenecer a la figura. Alrededor de la figura se han formado capas de calcita que podrían haber cubierto otros grabados -algunos trazos aislados son visibles entre este motivo y el siguiente-, pero, en todo caso, la parte inferior del caballo nunca fue representada. Por su forma parece corresponderse con el grabado n. ${ }^{\circ} 5$ de la fig. 3 de N. Casteret (1933).

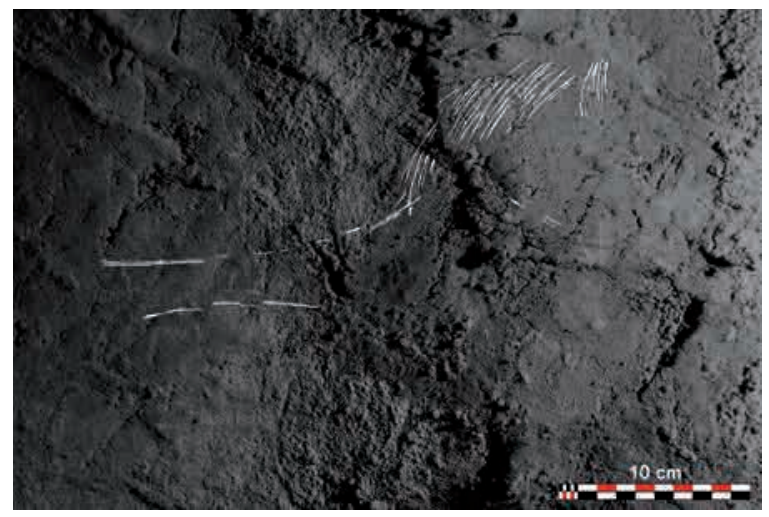

FIG. 6. Fotografía y calco del caballo A.1. 

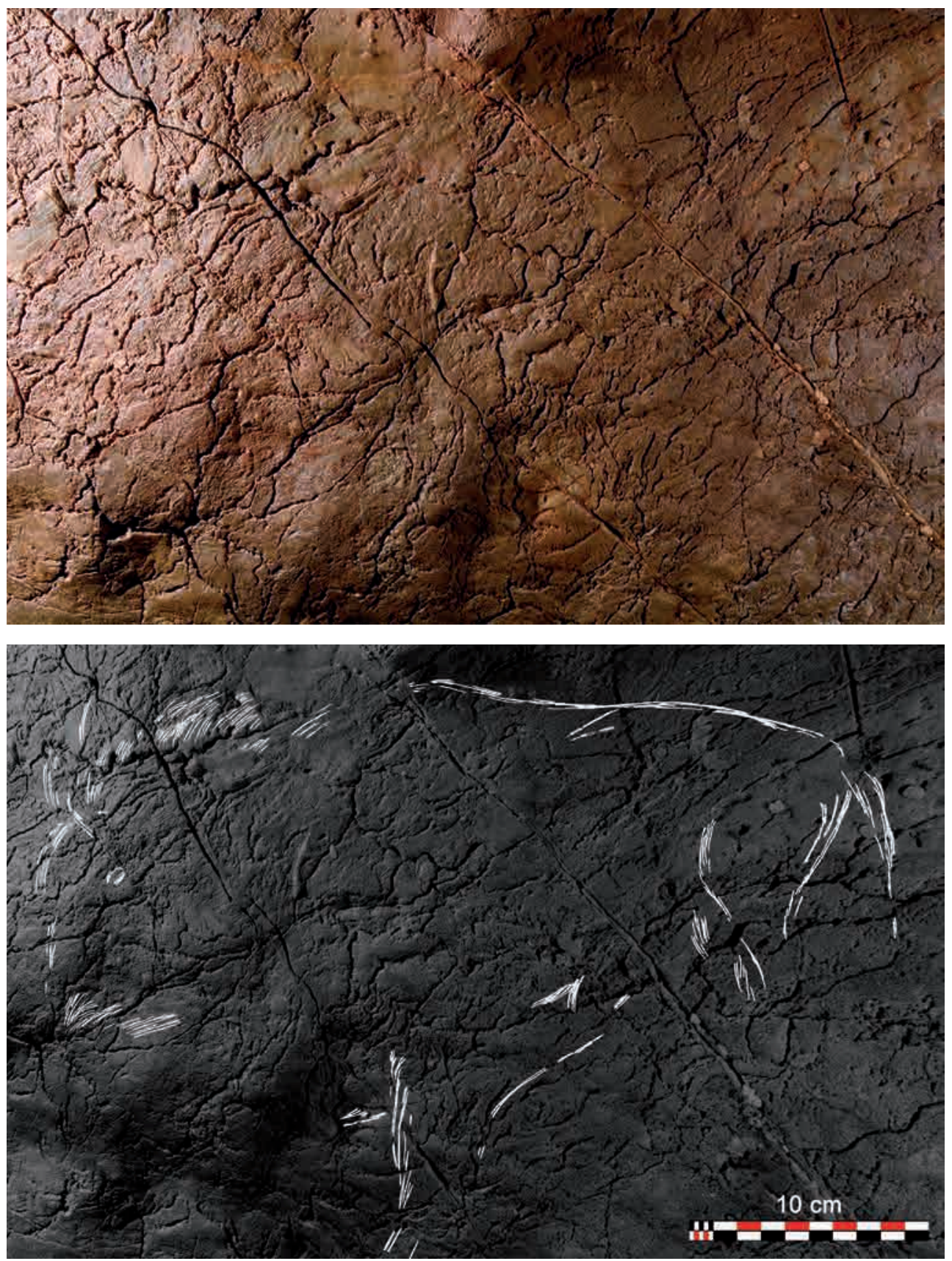

Fig. 7. Fotografía y calco del bisonte A.3 y del cuarto trasero A.2.

- Cuarto trasero (A.2) (Fig. 7). Dimensiones: $17 \times 6 \mathrm{~cm}$; altura al suelo: $51 \mathrm{~cm}$

Cuarto trasero de animal indeterminable, orientado a la izquierda y trazado mediante grabado fino múltiple. Se trata de una pata trasera y del arranque del vientre, sin que se haya representado ninguna otra parte anatómica. No podemos descartar que se trate de la pata delantera del siguiente bisonte, aunque entonces su tamaño resultaría desproporcionadamente mayor.

- Bisonte (a.3) (Fig. 7). Dimensiones: 36 x $22,5 \mathrm{~cm}$; altura al suelo: $54 \mathrm{~cm}$ 

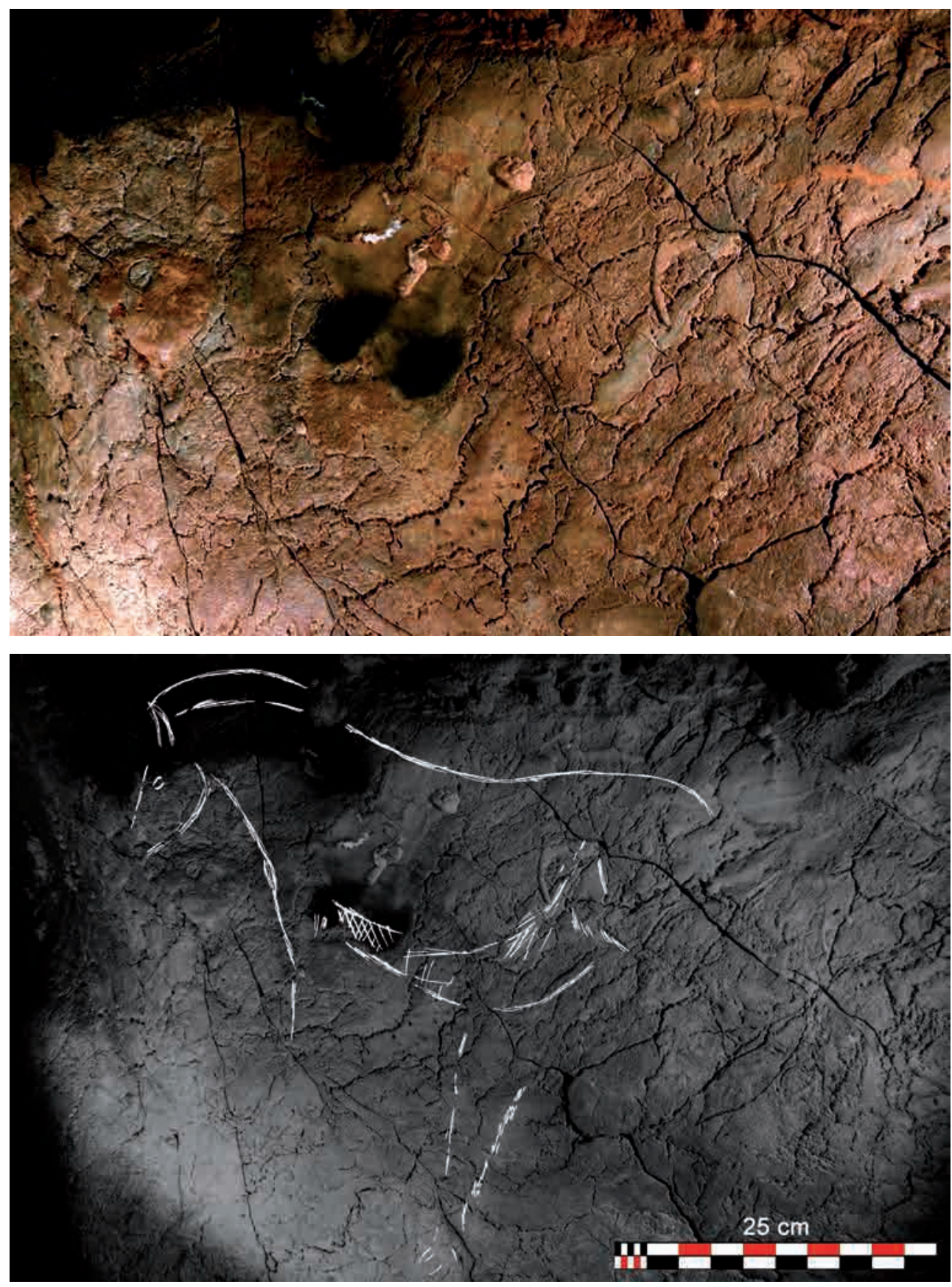

FIG. 8. Fotografía y calco del caballo A.5 y de la pata A.4.

Bisonte casi completo orientado hacia la izquierda y situado por encima de la figura anterior. Grabado con trazos poco profundos yuxtapuestos, se compone de la giba representada mediante tracitos curvos paralelos a modo de pelaje (Fig. 23f), dos cuernos, línea frontonasal formada por trazos cortos paralelos, el ojo, hocico, barba, las patas traseras en dos planos, la cola larga y el lomo con un despiece triangular en la cruz. El soporte es la roca caliza limpia sin casi formaciones de calcita, 


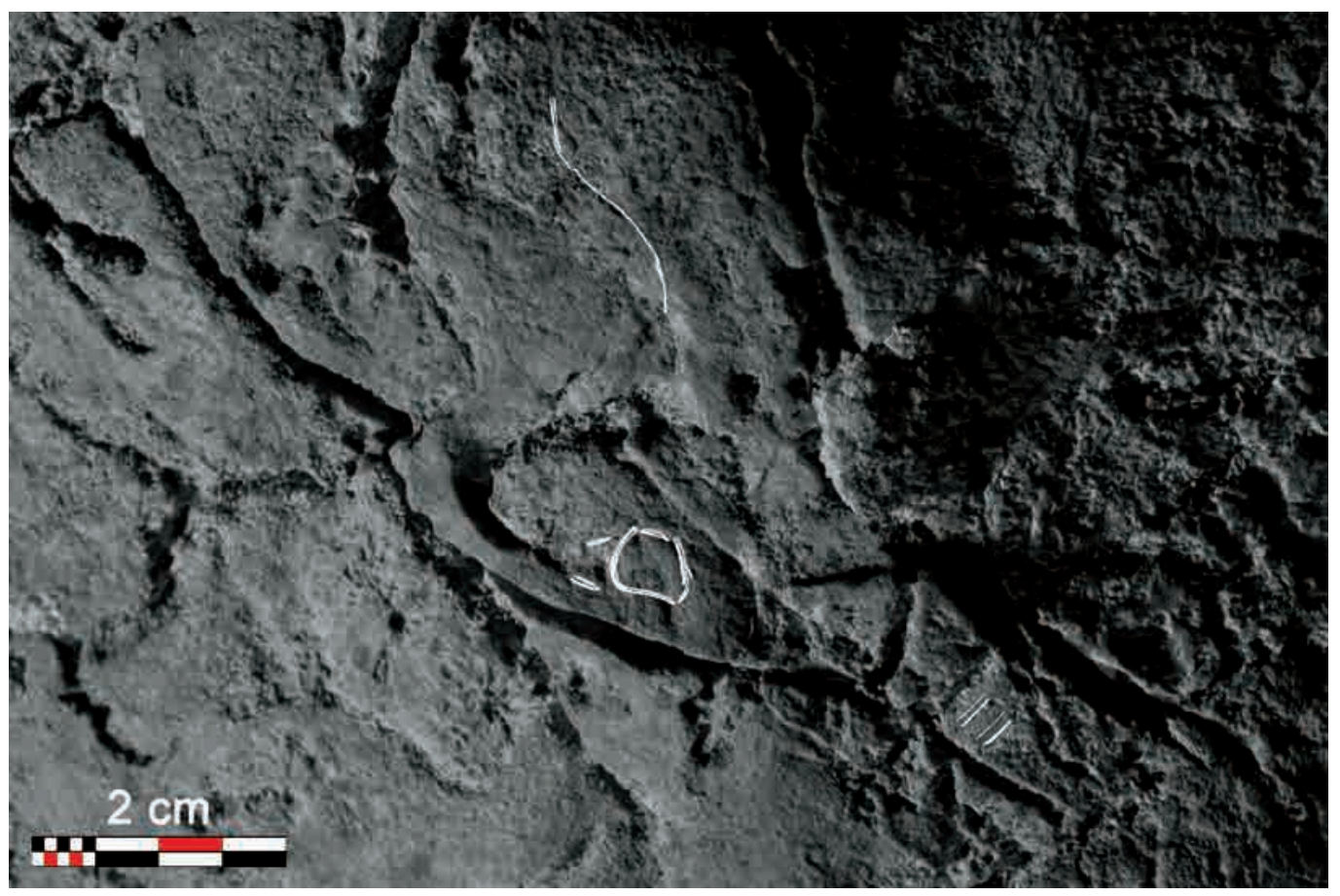

FIG. 9. Fotografía y calco del ojo y cuerno A.6.

pero con una película constante de agua en toda la superficie.

- Pata (A.4) (Fig. 8). Dimensiones: 23 x $5 \mathrm{~cm}$; altura al suelo: $35 \mathrm{~cm}$

Pata delantera de animal grabada mediante incisiones simples yuxtapuestas, con la pezuña bisulca detallada y trazos cortos a modo de pelaje. Por las proporciones y anatomía representada podría formar parte de una misma figura con el cuarto trasero A.2, aunque no se observan los puntos de unión.

- Caballo (A.5) (Fig. 8). Dimensiones: 44,5 x $25 \mathrm{~cm}$; altura al suelo: $71 \mathrm{~cm}$

Caballo prácticamente completo orientado a la izquierda, grabado sobre una pared muy alterada por manchas de carburo y por algunas formaciones calcíticas que afectan a los grabados. Se localiza inmediatamente antes del recodo que da al tramo final. El animal se ha trazado mediante grabado múltiple y se compone de doble crinera lineal, la cabeza afectada por las manchas de carburo, pero donde son visibles una oreja y el ojo además de la línea frontonasal y el maxilar, el pecho, el arranque de las patas delanteras, una doble línea de vientre con relleno interior a modo de trazos entrecruzados, el arranque de las patas traseras y la línea del lomo. El lugar correspondiente a la nalga y cola está ocupado por el tren delantero del bisonte anteriormente descrito, por lo que podemos deducir que esta figura fue ejecutada con posterioridad al bisonte A.3.

- Ojo y cuerno (a.6) (Fig. 9). Dimensiones: 6 x $2,5 \mathrm{~cm}$; altura al suelo: $74 \mathrm{~cm}$

Ojo ovalado con la comisura indicada orientado a la derecha y un cuerno de trazo único por encima. Se localiza en el recodo que da al tramo final. La pared está muy frotada por lo que es posible que la representación fuera más completa.

- Líneas (A.7) (Fig. 11). Dimensiones: 4 x $3 \mathrm{~cm}$; altura al suelo: $70 \mathrm{~cm}$

Dos series de líneas grabadas con trazo único pero formando dos surcos paralelos. Se sitúa entre la unidad anterior y por encima de la siguiente, en el recodo de la gatera. Podrían formar parte del lomo y grupa de la representación anterior.

- Bisonte (A.8) (Figs. 10 y 11). Dimensiones: $22,5 \times 10 \mathrm{~cm}$; altura al suelo: $65 \mathrm{~cm}$ 
Bisonte completo orientado hacia la izquierda grabado con incisión fina sobre la roca caliza. Presenta la giba indicada mediante trazos en forma de pelaje que continúa en la cabeza con dos cuernos sinuosos y el ojo señalado, hocico con boca, la barba, el arranque del tren delantero, el arranque de la pata trasera y del vientre, la nalga, el lomo y la cola. En el interior del tronco se observa una serie de líneas transversales a modo de pelaje de difícil interpretación (posible crinera y lomo de caballo) así como unos trazos curvilíneos sobre el hocico.

La figura se corresponde con el bisonte localizado por N. Casteret en 1930, en el recodo de la gatera, y no considerado de nuevo hasta la actualidad.



FIG. 10. Fotografía del bisonte A.8.

- Animal indeterminado (A.9) (Fig. 12). Dimensiones: 19 x $14 \mathrm{~cm}$; altura al suelo: $59 \mathrm{~cm}$

Trazos situados por debajo del bisonte anterior y grabados con incisiones poco profundas. Es probable que en origen representaran un animal que no resulta reconocible en la actualidad.

- Bisonte (A.10) (Fig. 12). Dimensiones: $60 \mathrm{x}$ $21 \mathrm{~cm}$; altura al suelo: $61 \mathrm{~cm}$

Bisonte orientado a la derecha una vez superado el recodo y ya en el tramo final de la gatera.
Únicamente se han grabado un cuerno, el ojo, el pelaje de la giba y la línea del lomo. Se trata de la zona más estrecha de la Galería, y las dificultades de movimiento son probablemente la causa de la factura incompleta de la figura.

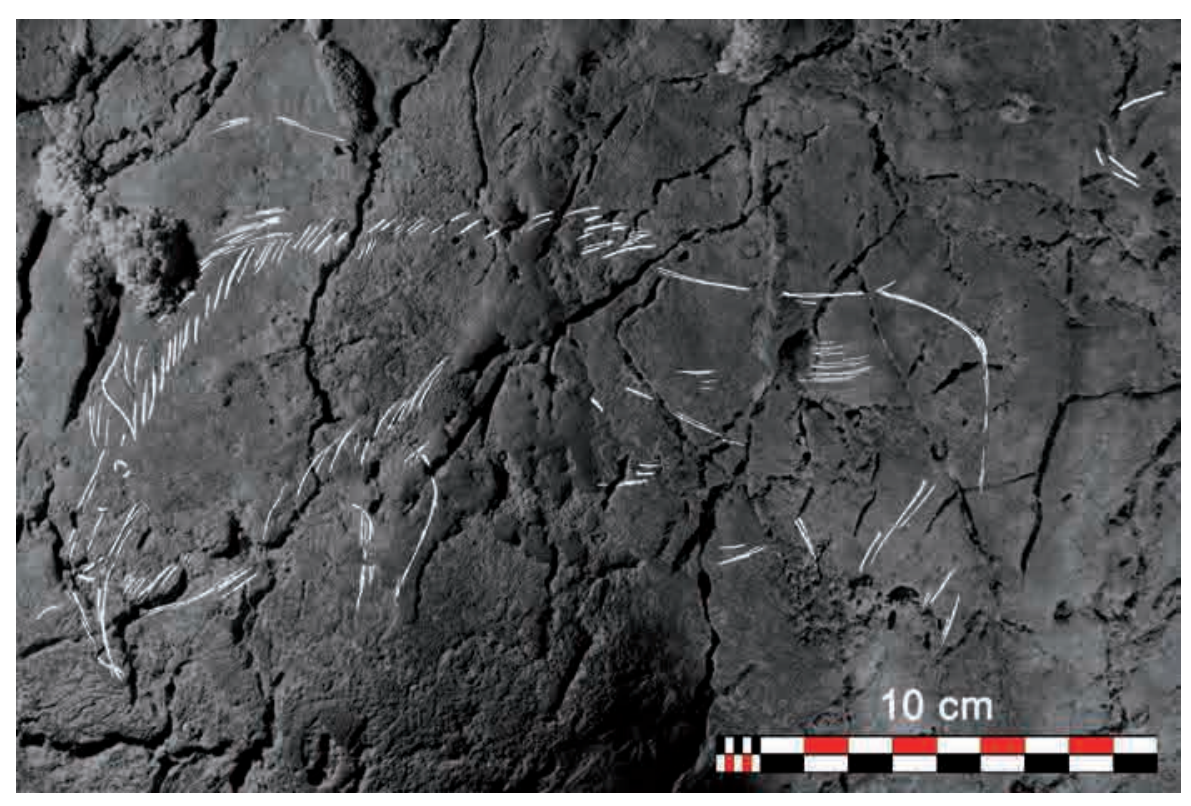

FIG. 11. Calco del bisonte A.8 y del motivo indeterminado A.7. 

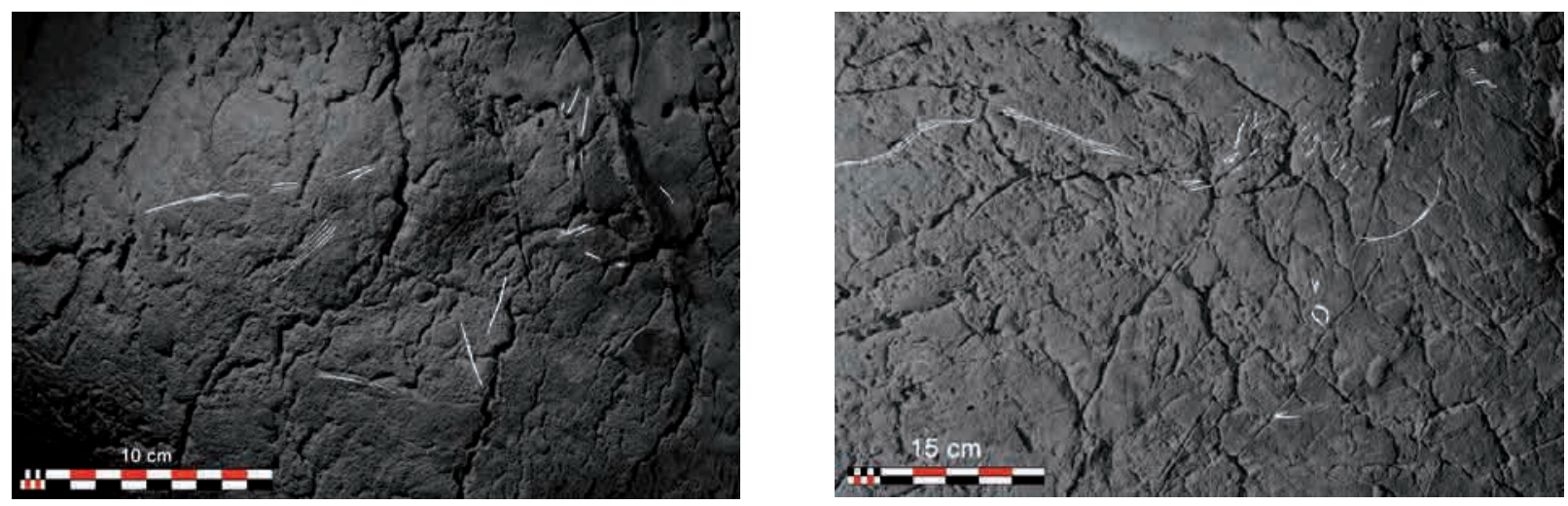

Fig. 12. Calco del motivo indeterminado A.9 y del bisonte A.10.

- Bisonte (A.11) (Fig. 13). Dimensiones: $27 \mathrm{x}$ $21 \mathrm{~cm}$; altura al suelo: $71 \mathrm{~cm}$

Bisonte orientado a la derecha situado por encima del anterior, después del recodo en el tramo

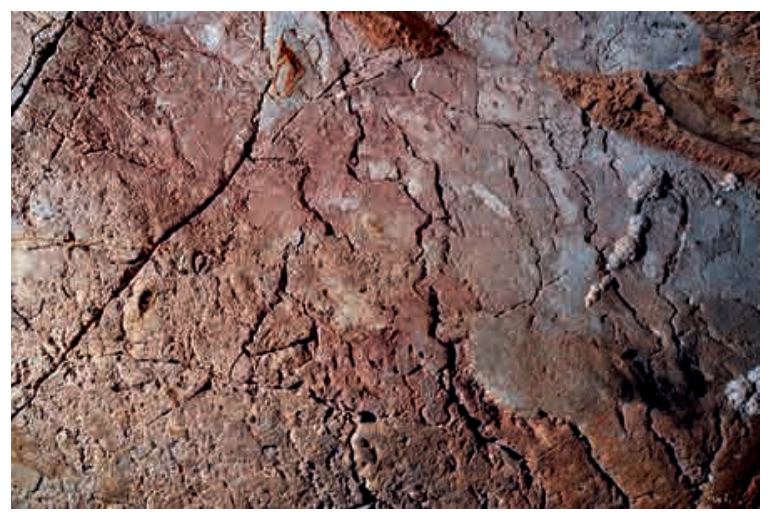

final de la gatera. Se reduce a la parte inferior del animal con indicación de la barba mediante trazos cortos, el arranque de las patas delanteras y la línea del vientre.

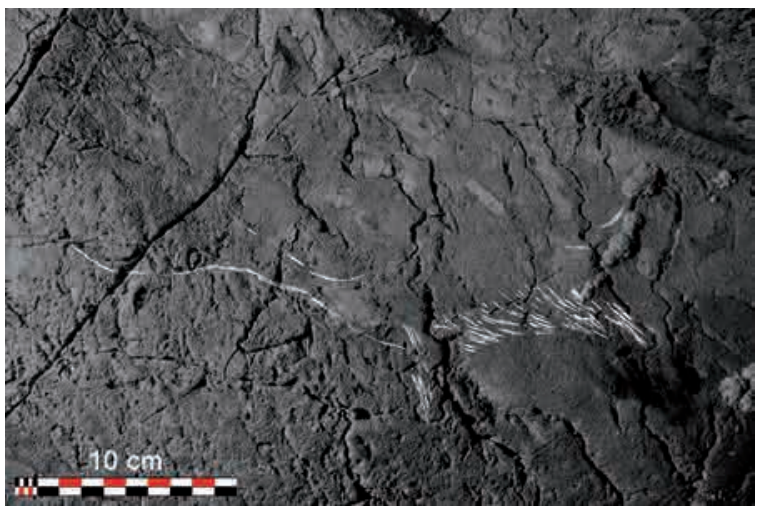

Fig. 13. Fotografía y calco del bisonte A.11.

\subsection{Pared izquierda (B)}

La pared izquierda es plana y húmeda en los primeros $2 \mathrm{~m}$ donde, después de una pequeña concavidad a ras de suelo, gira su orientación y enlaza con el tramo final de dimensiones muy reducidas.

- Línea (в.1) (Fig. 14). Dimensiones: 24 x 9 $\mathrm{cm}$; altura al suelo: $125 \mathrm{~cm}$

Línea curva grabada con trazo ancho y poco profundo, única situada a mitad del recorrido del primer tramo de la gatera. Por debajo se observa otra línea más o menos paralela y en el extremo izquierdo otras series de trazos. Aunque la formación de espeleotemas ha podido cubrir más trazos, no se identifican formas anatómicas concretas que nos permitan considerar el grabado como una representación figurativa zoomorfa.

- Bisonte (в.2) (Fig. 15). Dimensiones: 26 x 14 $\mathrm{cm}$; altura al suelo: $88 \mathrm{~cm}$

Representación parcial de bisonte orientado hacia la derecha. El lomo y giba se acomodan al límite de una pequeña hornacina a la altura del 

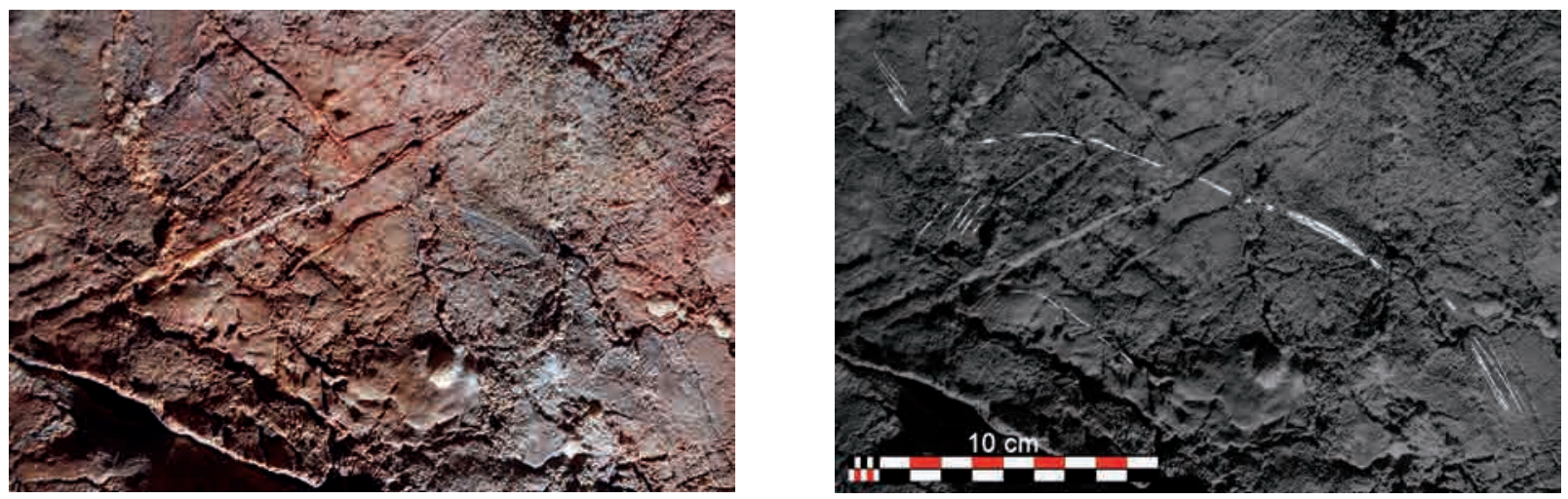

Fig. 14. Fotografía y calco de la línea B.1.

suelo. Otros trazos grabados sugieren el tren anterior y pelaje del animal, aunque la pared caliza está recubierta por formaciones más y menos gruesas

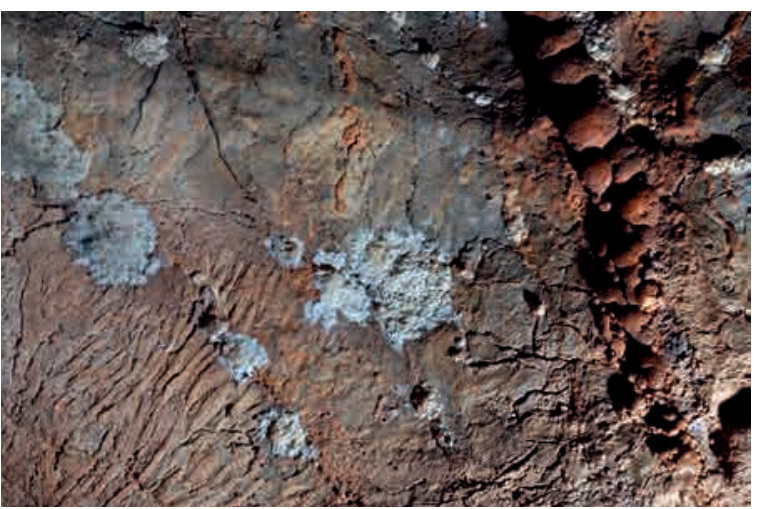

de calcita que, a su vez, han tapado parte de los grabados, siendo muy difícil su interpretación en el estado actual.

FIG. 15. Fotografía y calco del bisonte B.2.

- Caballo (в.3) (Fig. 16). Dimensiones: 28 x 21 $\mathrm{cm}$ : altura al suelo: $61 \mathrm{~cm}$

Caballo orientado hacia la derecha sobre una convexidad de la pared frotada, con manchas de carburo y con formaciones de calcita, que dificultan enormemente su lectura. Se observa el trazado de la línea frontal, del hocico con nariz y despiece, boca, el pecho, el ojo y una oreja, la crinera, formada por trazos cortos paralelos y por dos líneas, y el lomo. Es posible que la figura se representara más completa y que algunas partes anatómicas resulten imperceptibles debido a la profunda alteración del soporte.

(C) Universidad de Salamanca

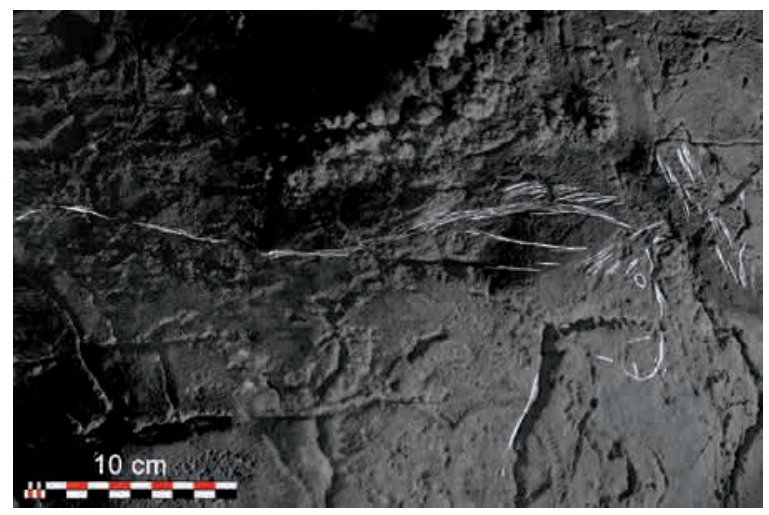

FIG. 16. Calco del caballo B.3.

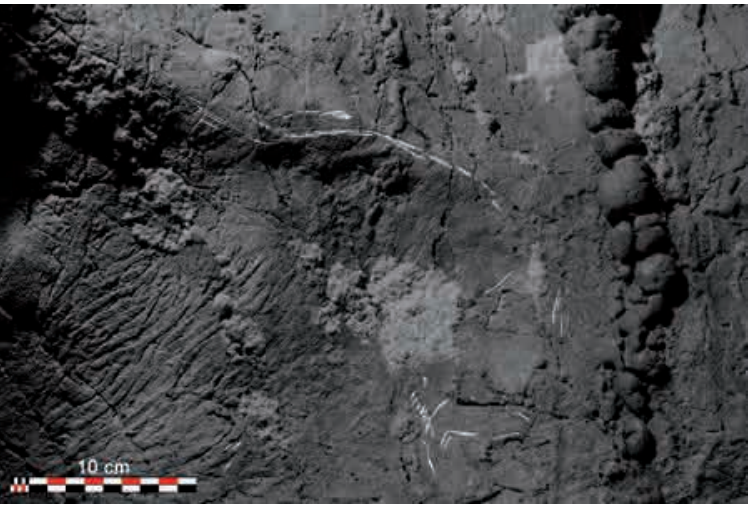



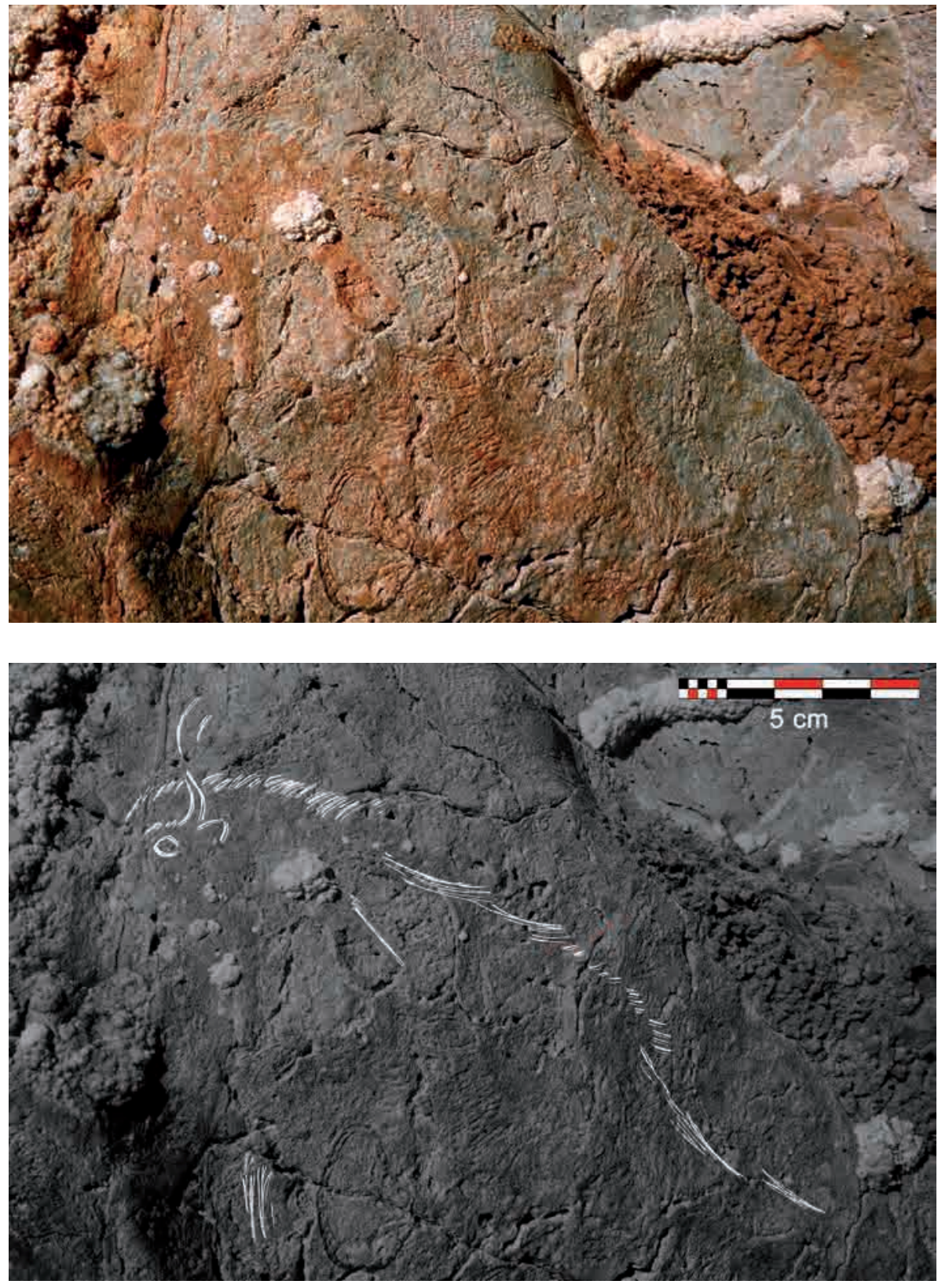

FIG. 17. Fotografía y calco del bisonte B.4.

la figura, en su parte inferior, se observan trazos aislados. Se superponen a los trazos grabados restos de ocre en la zona de la giba.

- Bisonte (в.5) (Fig. 18). Dimensiones: $67 \mathrm{x}$ $29 \mathrm{~cm}$; altura al suelo: $63 \mathrm{~cm}$

Bisonte completo orientado hacia la derecha. Mediante grabado poco profundo y de múltiples pasadas se han representado la cabeza con los dos cuernos en perspectiva, el ojo con despiece linear y la oreja, el hocico con la nariz y la lengua indicadas, el pelaje de la frente y de la giba, el lomo con doble línea, la cola larga y las patas traseras con las extremidades en primer plano adelantadas y el corvejón y la pezuña indicadas. En el vientre unos trazos en el arranque señalan el sexo y tres largas líneas verticales atraviesan el cuerpo del animal, pudiendo simular proyectiles que abaten al animal. Las patas delanteras solamente han sido sugeridas y una serie de trazos cortos y oblicuos señalan la barba. Dos puntos rojos se yuxtaponen

- Bisonte (в.4) (Fig. 17). Dimensiones: 15,5 x $5 \mathrm{~cm}$; altura al suelo: $76 \mathrm{~cm}$

Representación parcial de bisonte orientado hacia la izquierda. Se ha grabado la giba a base de trazos cortos paralelos y líneas, los dos cuernos en perspectiva, el ojo con despiece a modo de tracitos cortos, una oreja y la línea del lomo. Alrededor de al ojo y a la giba del bisonte. Se trata de la figura de mayor tamaño y con mayor cantidad de detalles anatómicos representados.

- Puntos rojos (в.6) (Fig. 18). Dimensiones: 2 x $2 \mathrm{~cm}$; altura al suelo: 66 y $63 \mathrm{~cm}$ 

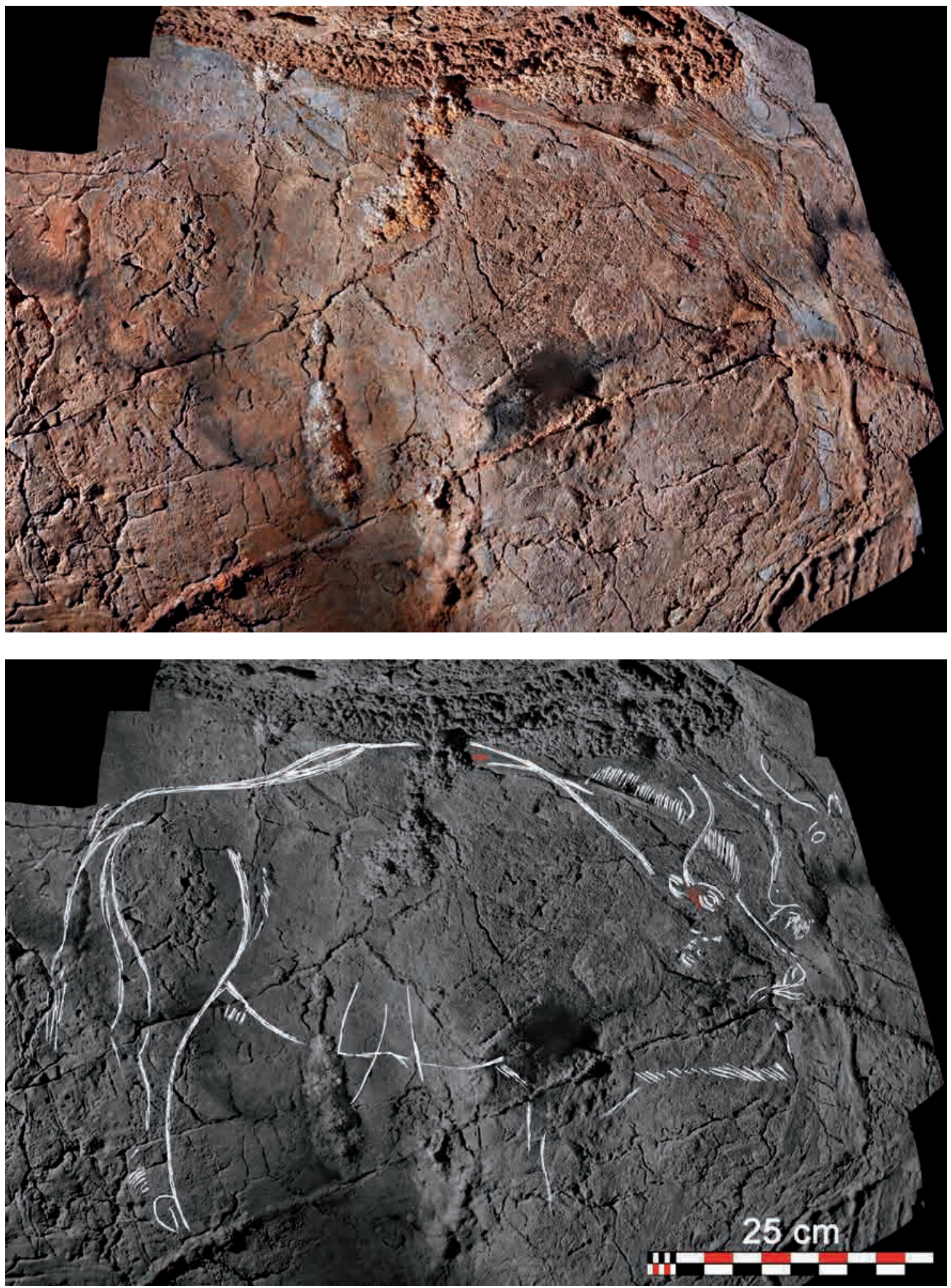

Fig. 18. Ortofotografía y calco de los bisontes B. 5 y B. 7 y de los puntos rojos B. 6 . 


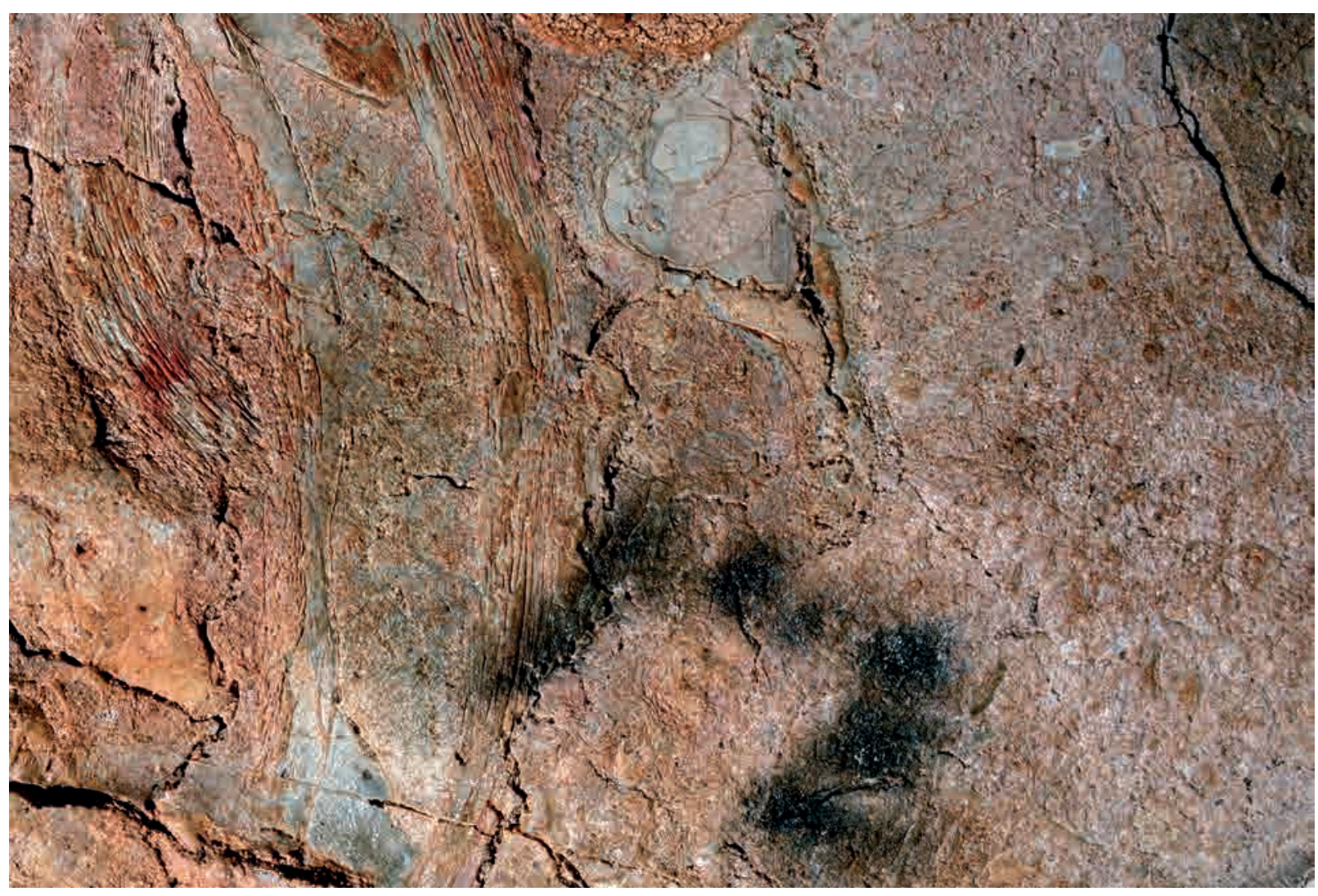

Fig. 19. Fotografía del bisonte B.7.

Dos puntos rojos aplicados digitalmente situados junto al ojo y junto al lomo del bisonte anterior.

- Bisonte (в.7) (Figs. 18 y 19). Dimensiones: $12 \times 16 \mathrm{~cm}$; altura al suelo: $67 \mathrm{~cm}$



Cabeza de bisonte orientada hacia la izquierda y enfrentada a la anterior en una amplia concavidad del tramo final de la gatera. Se han grabado finamente los dos cuernos en perspectiva, el ojo con despiece linear indicado, la oreja y el hocico

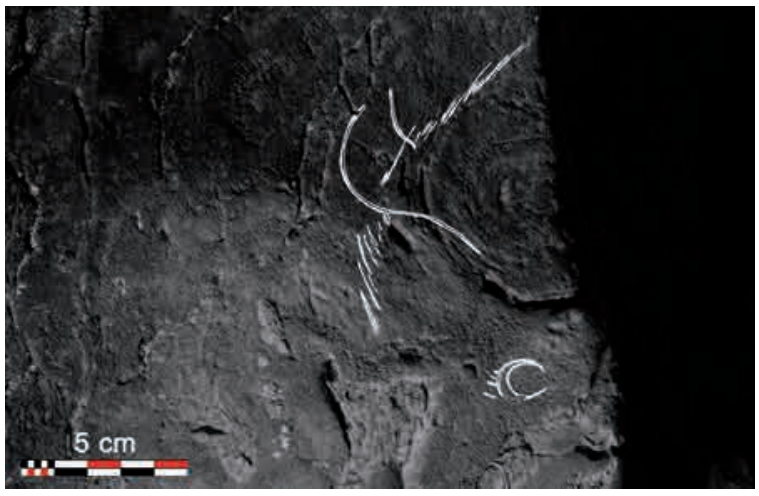

FIG. 20. Fotografía y calco del bisonte B.8. 

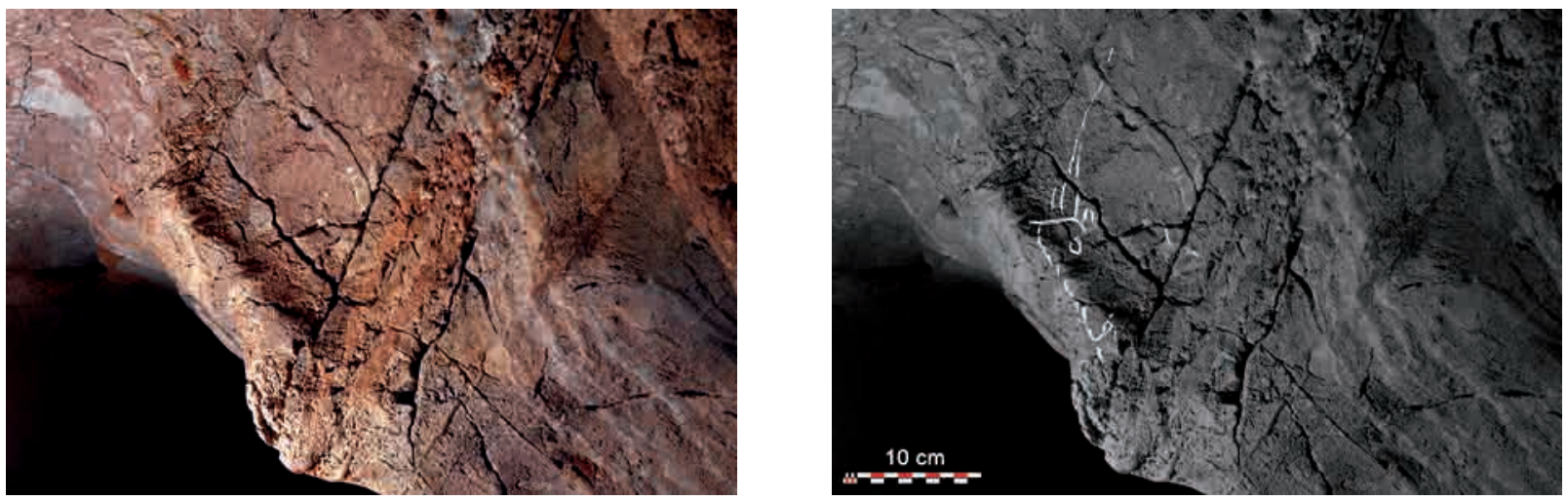

FIG. 21. Fotografía y calco del bisonte B.9.

con la lengua representada y la barba. Una mancha de carburo afecta a la figura.

- Bisonte (в.8) (Fig. 20). Dimensiones: 16 x 11 $\mathrm{cm}$; altura al suelo: $57 \mathrm{~cm}$

Cabeza de bisonte orientada hacia la izquierda en un saliente entre dos concavidades del tramo final de la gatera. Presenta los dos cuernos en perspectiva, el ojo con la comisura indicada y varios trazos cortos que representan la línea del frontal y el arranque de la giba.

- Bisonte (в.9) (Fig. 21). Dimensiones: 22 x 18 $\mathrm{cm}$; altura al suelo: $70 \mathrm{~cm}$

Cabeza de bisonte orientada hacia la izquierda en una leve concavidad en el tramo final de la gatera, prácticamente intransitable. Finamente grabada, se identifican un cuerno sinuoso, la oreja, el ojo, la línea frontonasal formada por tracitos cortos, el hocico con la lengua indicada y el arranque de la giba.

\section{Síntesis de los grabados de la 'Galería de los Bisontes'}

El nuevo sector decorado descubierto en la cueva de Alkerdi se caracteriza por sus exiguas dimensiones resultando casi impracticable (Fig. 22) $y$, aparentemente, poco apto para contener representaciones parietales paleolíticas, por lo menos en la densidad señalada, con 20 motivos en apenas $3 \mathrm{~m}$ de desarrollo (Figs. 24 y 25). Aun así, existen en el registro paleolítico otros casos similares en los que contrasta lo reducido y aparentemente inapropiado del espacio con la gran cantidad de figuras representadas. Así sucede, por ejemplo, en las cuevas de Fronsac o la galería terminal de Font-de-Gaume en

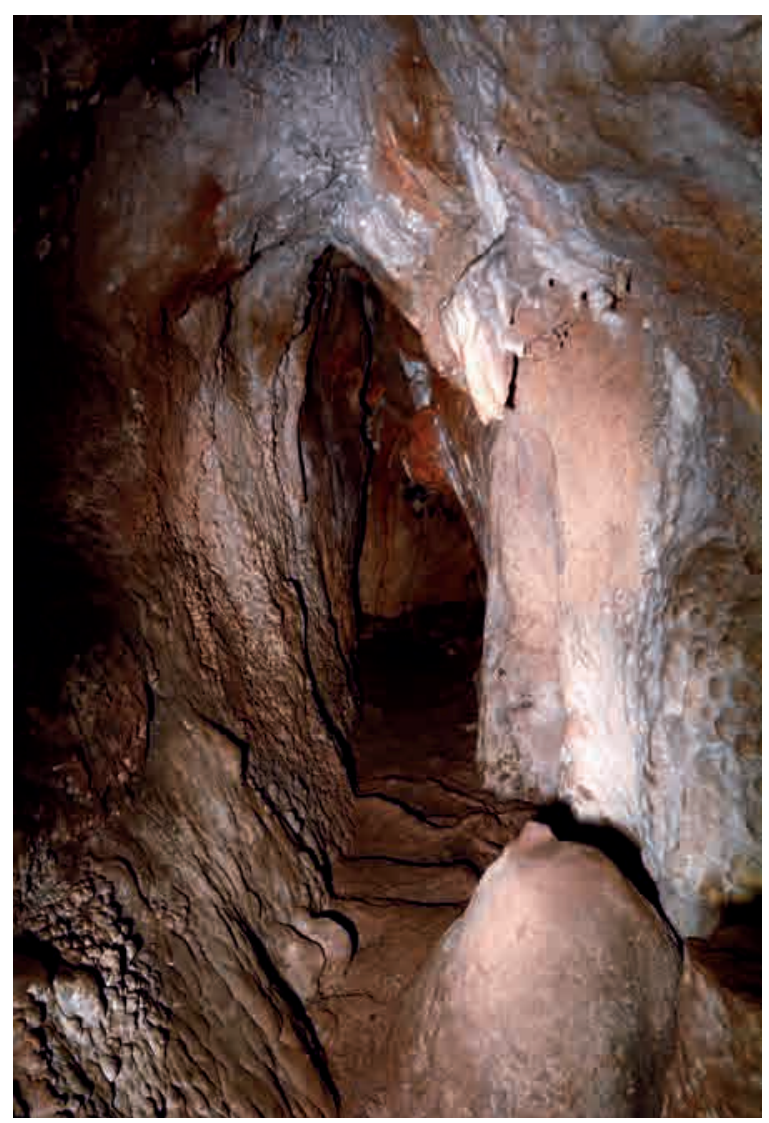

Fig. 22. Tramo de la galería donde se concentran los motivos parietales. 

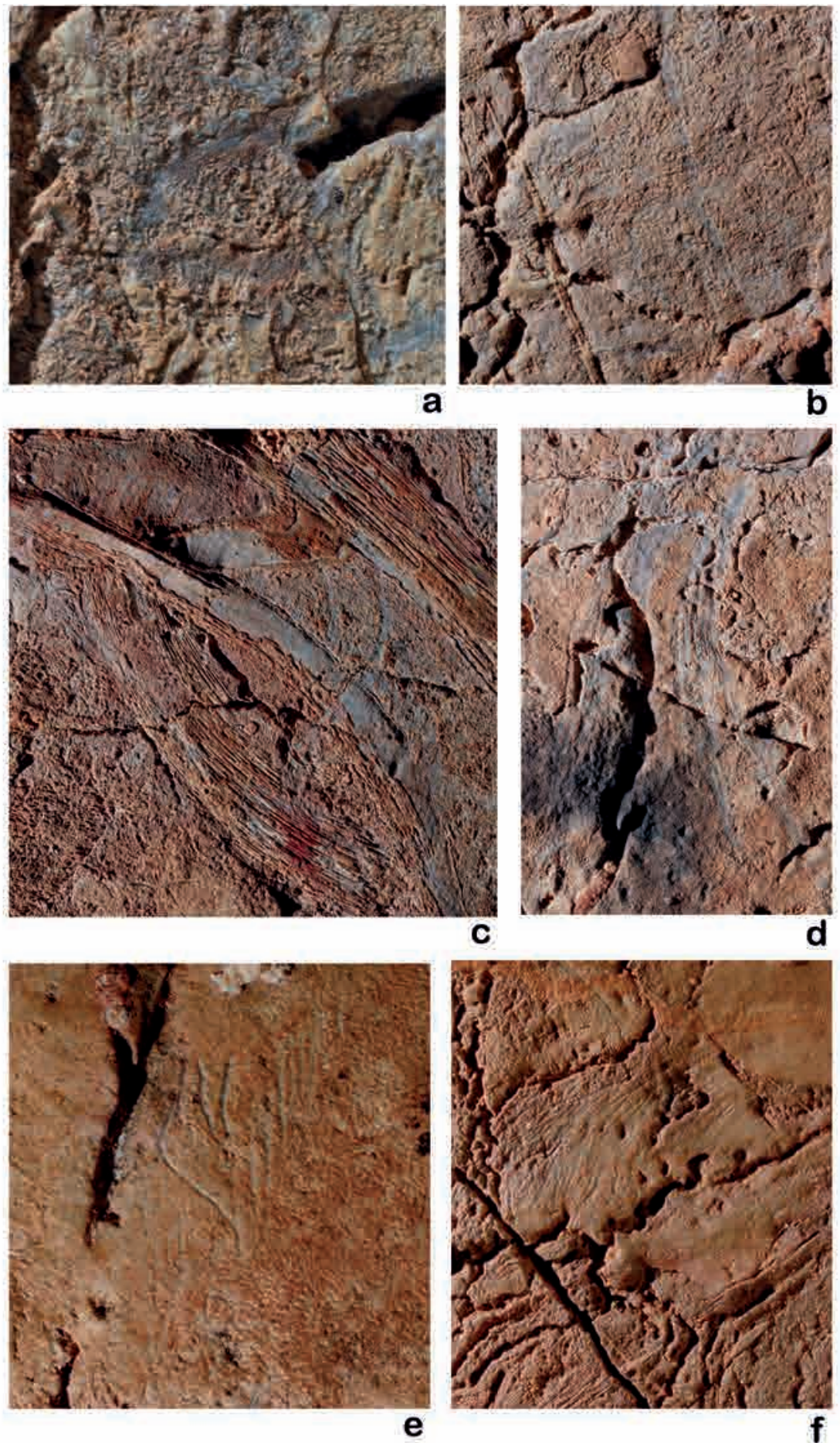

FIG. 23. Detalles de las figuras en la 'Galería de los Bisontes'; a) ojo del bisonte B.9; b) pezuña bisulca con pelaje del bisonte completo B.5; c) cornamenta, pelaje de la frente y la giba, ojo y oreja del bisonte $B .5$; d) detalle de la cola y linea de las nalgas del bisonte B. 5 ; e) bisonte A.8, detalle del ojo, cornamenta y pelaje de la giba; f) pelaje de la giba del bisonte A.3. Pueden apreciarse en todos los casos las caracteristicas técnicas y formales reseñadas. 


\section{PARED DERECHA}

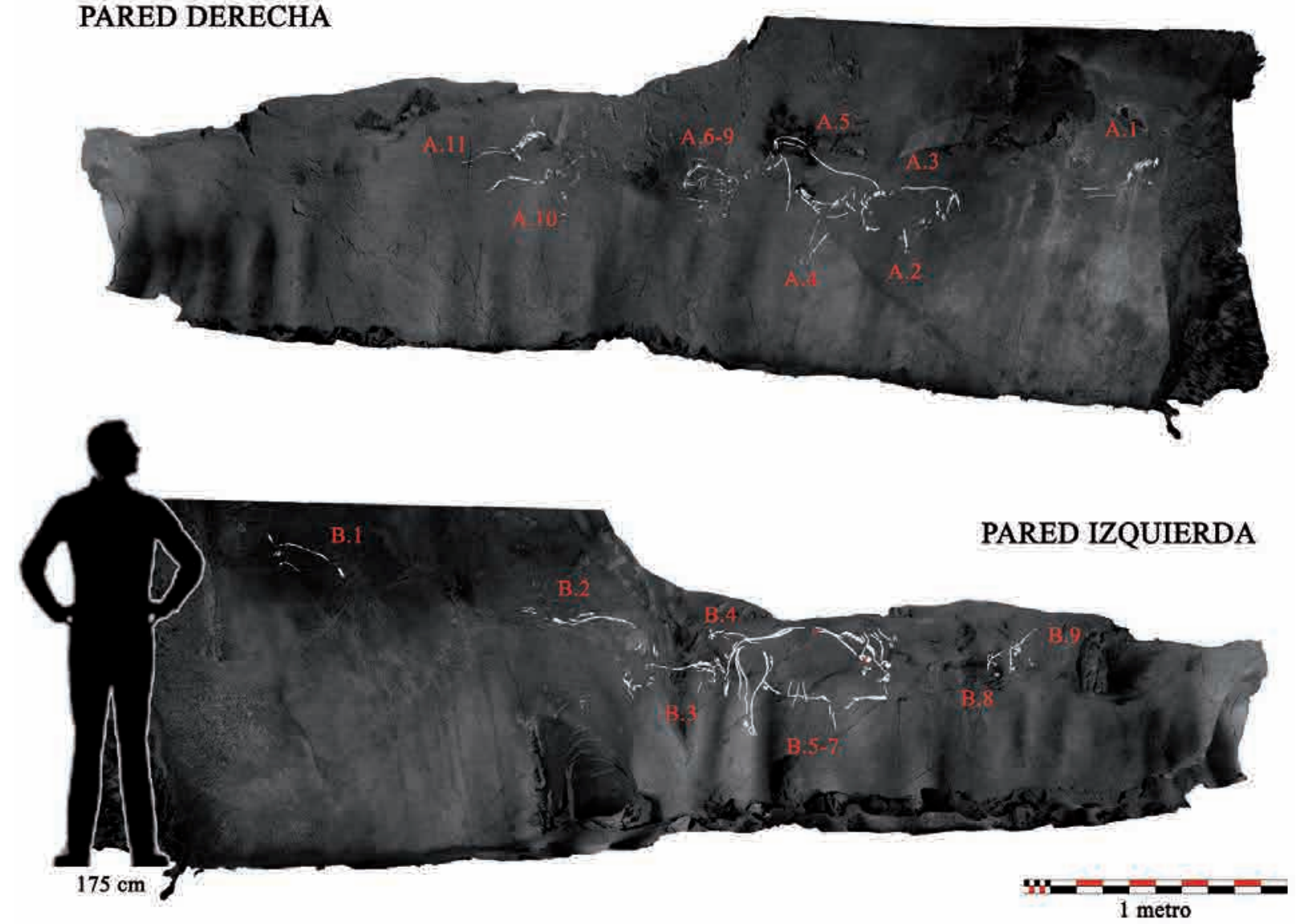

Fig. 24. Vista ortofotográfica de las dos paredes decoradas de la 'Galería de los Bisontes' con la situación de los grabados indicados mediante los calcos.

la Dordońa, en la galerie Breuil de Mas d'Azil o en la fissure ornée de Etxeberri en los Pirineos, o en la Cola de Caballo de Altamira y en la diaclasa de los signos situada al fondo de Pasiega A, en la región cantábrica.

Las representaciones descubiertas en la 'Galería de los Bisontes' poseen unas características muy homogéneas desde el punto de vista técnico y formal. Técnicamente, las figuras han sido realizadas mediante incisiones poco profundas, probablemente como consecuencia de la dureza del soporte, yuxtapuestas formando haces en lugar de surcos profundos y en muchas ocasiones fruto de una única pasada del útil. La buena conservación de los trazos en la galería permite ver el grabado sin que apenas exista pátina, destacándose en gris sobre el fondo rojizo de la caliza. Este efecto debía ser más evidente en el momento de realizar las figuras, lo que permitiría verlas fácilmente sin necesidad de efectuar surcos profundos (Fig. 23).

La tipometría de las figuras, en su mayor parte de muy pequeño tamaño, está igualmente ligada a las características del espacio decorado: las exiguas dimensiones de la gatera impiden la realización de figuras grandes, especialmente en su tramo final, donde la dificultad de movimiento debió de ser considerable para el grabador. Aun así, resulta sorprende el formato completo y tamaño considerable del bisonte в. 5 que, además, cuenta con numerosos detalles en su representación. Para poder trazarlo, el artista debió de recostarse pegado a la pared opuesta y difícilmente podía observar la figura completa en el momento de realizarlo. 


\begin{tabular}{|c|c|c|c|c|c|c|c|c|c|}
\hline & Bisonte & Caballo & Ciervo & Cierva & Lobo & $\begin{array}{c}\text { Animal } \\
\text { indet. }\end{array}$ & Líneas & $\begin{array}{c}\text { Mancha } \\
\text { roja }\end{array}$ & TotaL \\
\hline A & 1 & $1-1 ?$ & 1 & 0 & 0 & 1 & 3 & 0 & $7-1 ?$ \\
\hline B & 0 & 0 & 0 & 0 & 0 & 1 & 0 & 0 & 1 \\
\hline C & 0 & 0 & 0 & $1 ?$ & $1 ?$ & 0 & 0 & 0 & $2 ?$ \\
\hline D & 10 & 3 & 0 & 0 & 0 & 4 & 2 & 1 & 20 \\
\hline TотаL & 11 & $4-1 ?$ & 1 & $1 ?$ & $1 ?$ & 6 & 5 & 1 & $28-3 ?$ \\
\hline
\end{tabular}

Fig. 25. Cómputo total de motivos parietales distribuidos por sectores decorados (recuentos en los sectores A y C a partir de Barandiarán, 1974; recuentos en los sectores B y D a partir del estudio actual).

Por lo que respecta a las características formales, los bisontes, mayoritarios, poseen unos rasgos muy marcados y uniformes: cuernos en doble curvatura $-s-$, realizados mediante una única línea; representación de la barba y de la giba mediante trazos paralelos a modo de pelaje; indicación de los órganos sensoriales -ojo, oreja, boca-, en ocasiones con despieces asociados, y otros detalles anatómicos como la lengua, pezuñas, corvejón, sexo, etc. Se trata de figuras muy detalladas (Figs. 24 y 26), que siguen los convencionalismos propios del Magdaleniense medio pirenaico (Rivero, 2009).

En el caso de los caballos, menos numerosos, sus características se asocian igualmente a las representaciones de este período; crineras representadas mediante trazos paralelos y abundancia de detalles anatómicos tales como ojo, oreja, boca y despieces.

\section{Una nueva visión del arte parietal de la cueva}

El descubrimiento de los grabados de la 'Galería de los Bisontes' resulta de suma importancia ya que modifica de manera sustancial nuestro conocimiento sobre el arte parietal paleolítico de la cueva de Alkerdi.

Por un lado, la cantidad de representaciones contenidas en la cavidad ha aumentado exponencialmente, pasando las grafías animales de 7 seguras a un total de 24 (Fig. 25). Entre los temas seleccionados cabe destacar el dominio de bisontes, que alcanzan los 11 ejemplares. En total son 31 las grafías diferenciadas en el conjunto de la cueva, 3 de ellas de identificación dudosa.
Por otro lado, las convenciones gráficas y los recursos técnicos aplicados en los grabados de la 'Galería de los Bisontes' se diferencian de los conocidos en el mogote de calcita del extremo opuesto de la cavidad, descubierto por N. Casteret (1933) y estudiados por I. Barandiarán (1974). Dichas diferencias nos permiten plantear la hipótesis de dos fases distintas de decoración de la cavidad, no necesariamente muy alejadas en el tiempo.

Algunas de estas diferencias son el distinto acabado a nivel formal de las figuras; el bisonte grabado en el sector a difiere de los presentes en la 'Galería de los Bisontes' en el tratamiento de la giba -lineal y no mediante tracitos cortos- o en la representación de las pezuñas, al igual que en el caso del caballo. La presencia de un ciervo con relleno de trazos a modo de grabado estriado es también un aspecto reseñable que diferencia técnicamente ambos conjuntos. En cuanto a la temática, el primer sector presenta una diversidad de especies animales representadas mientras que en el segundo el dominio de los bisontes es absoluto.

\section{Alkerdi y los contactos interregionales durante el Magdaleniense medio}

Los datos arriba presentados enriquecen sustancialmente el conocimiento hasta ahora disponible del arte parietal de la cueva de Alkerdi y además vienen a completar el mapa conocido de las relaciones interregionales durante el período Magdaleniense.

La cueva se sitúa en el eje de relaciones Cantábrico-Pirineos, en proximidad de conjuntos tales como Altxerri o las cuevas de la colina de Gaztelu -Isturitz, Oxocelhaya y Erberua-, con los que guarda una fuerte relación. Además, se trata de un área que ha sido revalorizada en función de los recientes hallazgos y revisiones de conjuntos (Garate et al., 2015). 

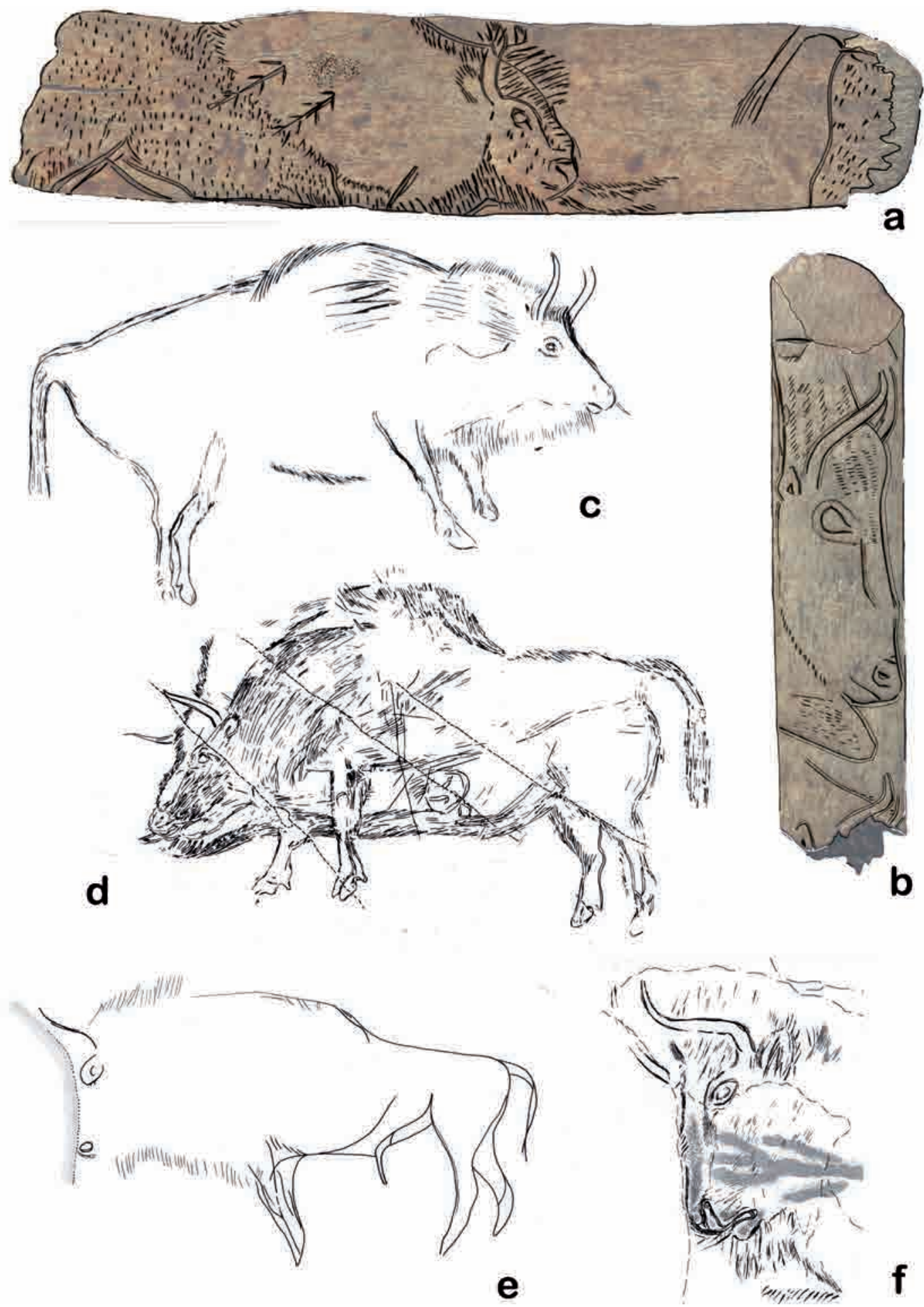

Fig. 26. Representaciones de bisontes del Magdaleniense medio cántabro-pirenaico: a-b) Isturitz (Rivero, 2009); c) Les Trois Frères (Bégouën y Breuil, 1958); d) Fontanet (Vialou, 1986); e) Altxerri (Altuna y Apellániz, 1976); f) Marsoulas (Fritz y Tosello, 1999). 
Con el primero, podemos destacar que ambos conjuntos se encuentran dominados por la figura del bisonte, aspecto que hay que vincular a los sitios pirenaicos tales como Niaux, Fontanet, Marsoulas, Les Trois-Frères o Le Tuc d'Audoubert (Ruiz Redondo, 2014).

Los paralelismos con estos yacimientos no se limitan a la temática representada, sino que atañen también a las convenciones formales. Como reseńábamos anteriormente, las particularidades de las figuras de los bisontes, en especial la abundancia de detalles anatómicos y la representación del pelaje del animal mediante trazos cortos paralelos, los vinculan a las figuras del Magdaleniense medio pirenaico. Se trata de convenciones que pertenecen al llamado morfotipo "Niaux", según el análisis realizado por J. Fortea et al. (2004). En un estudio reciente, el análisis estadístico de las características formales de los bisontes magdalenienses mobiliares y parietales de la Región Cantábrica, de los Pirineos y de la Dordońa ha puesto de manifiesto que la presencia de abundantes detalles anatómicos, la representación del pelaje o la lengua, es una característica de las representaciones pirenaicas por oposición al carácter lineal y la ausencia de órganos sensoriales de los bisontes cantábricos y de la Dordoña ${ }^{4}$ (Fig. 26). En este sentido, resulta particularmente interesante que en la misma cavidad se encuentren ambos tipos de figuraciones de bisontes, ya que el bisonte grabado del sector A, por su carácter lineal, se encuentra más próximo al resto de representaciones cantábricas, mientras que en la 'Galería de los Bisontes' las características son propias de las pirenaicas.

Otro aspecto que vincula el conjunto con aquellos conocidos en el Magdaleniense medio de los Pirineos es la presencia de una figura que puede interpretarse como 'herida' -bisonte в. 5-. Los bisontes heridos son un tema específico representado fundamentalmente en el arte mobiliar y parietal pirenaico, con algunos ejemplos destacables como las representaciones mobiliares de Isturitz (Fig. 26a) o parietales de Les Trois-Frères (Fig. 26c), Niaux o Fontanet (Fig. 26d).

En síntesis, los datos aportados contribuyen a dar una nueva visión no sólo del conjunto, hasta

${ }^{4}$ Sauvet, G. y Rivero, O. (2015): “D’un support à l'autre: l'art pariétal à la lumière de l'art mobilier", Paléo, 26, en prensa.

(C) Universidad de Salamanca ahora considerado como menor, de la cueva del Alkerdi, sino que vienen a enriquecer el panorama de los contactos a media y larga distancia durante el Magdaleniense medio. El yacimiento viene así a inscribirse en la corriente de intercambios culturales que recorre durante este período la Región Cantábrica y los Pirineos, constituyendo ambas regiones una unidad, con matices internos, de la que el arte parietal de la cueva de Alkerdi es un claro ejemplo.

\section{Bibliografía}

Altuna, J. y Apellániz, J. M. (1976): “Las figuras rupestres paleolíticas de la cueva de Altxerri (Guipúzcoa)", Munibe, 28, pp. 1-241.

Barandiarán, I. (1974): "Arte paleolítico en Navarra. Las cuevas de Urdax”, Príncipe de Viana, 134-135, pp. 9-47.

Barandiarán, I. (1977): "Prospecciones Arqueológicas en Sorgiñen-Leze (Zugarramurdi-Navarra)", Príncipe de Viana, 148/149, pp. 349-369.

Barandiarán, I. y Cava, A. (2008): "La ocupación gravetiense de la cueva de Alkerdi (Urdax)". En La Tierra te sea leve. Arqueología de la muerte en Navarra. Pamplona: Gob. de Navarra, pp. 43-45.

Barandiarán, I.; Cava, A. y Elorrieta, I. (2010): “Alternancia/complementariedad en las cuevas de Alkerdi y Berroberría”, Cuadernos de Navarra, 18, pp. 9-40.

Barandiarán, J. M. dE (1946): "Catalogue des stations préhistoriques des Pyrénées basques”, Ikuska, 1, pp. 24-40.

Barandiarán, J. M. DE (2005): Diario personal. Volumen I (1817-1936). Desde los primeros trabajos cientificos hasta el inicio del exilio. Col. Sara, 6, t. II. Ataun: Fundación José Miguel de Barandiarán.

Barandiarán, L. (1989): Cartas a José Miguel de Barandiarán (primera etapa, 1915-1936). Donostia/San Sebastián: Sociedad guipuzcoana de Ediciones y Publicaciones.

BégouëN, H. y Breuil, H. (1958): Les cavernes du Volp : Trois Frères, Tuc d'Audoubert. Col. Travaux de l'Institut de Paléontologie Humaine. Paris: Arts et métiers graphiques.

Casteret, N. (1933): "Une nouvelle grotte à gravures dans les Pyrénées. La grotte d'Alquerdi”. En XVe Congrès International d'Anthropologie et d'Archéologie Préhistorique. (Paris, 1931). Paris, pp. 384-389.

Cava, A.; Elorrieta, I. y Barandiarán, I. (2009): “El Gravetiense de la cueva de Alkerdi (Urdax, Navarra): análisis y contexto de su industria lítica”, Munibe (Antropologia-Arkeologia), 60, pp. 51-80.

Fortea, J.; Fritz, C.; García, M.; SANChidrián, J. L.; Sauvet, G. y Tosello, G. (2004): "L'art pariétal paléolithique à l'épreuve du style et du carbone-14". En Oтte, M. (dir.): La spiritualité. Actes du Colloque 
de la Commission 8 de l'UISPP (Paléolithique supérieur), 2003. ERAUL, 106. Liège, pp. 163-175.

Fritz, C. y Tosello, G. (1999): "Nouveau regard sur la grotte ornée de Marsoulas", Bulletin de la Société Préhistorique de l'Ariège, LIV, pp. 83-115.

Fritz, C. y Tosello, G. (2007): "The hidden meaning of forms: methods of recording Paleolithic parietal art", Journal of Archaeological Method and Theory, 14 (2), pp. 48-80. http://dx.doi.org/10.1007/s10816-007-9027-3

Garate, D.; Rivero, O.; Ruiz-Redondo, A. y Rios-GaRAIZAR, J. (2015): "At the crossroad: A new approach to the Upper Paleolithic art in the Western Pyrenees", Quaternary International, 364, pp. 283-293. http://dx.doi.org/10.1016/j.quaint.2014.05.054
Loriana, M. DE (1940): "Excavaciones arqueológicas en la gruta y covacho de Berroberría, término de Urdax (Navarra) y sus inmediaciones", Atlantis, xv, pp. 91-102.

Maluquer de Motes, J. (1965): "La estratigrafía del covacho de Berroberría (Urdax, Navarra)", Miscelánea en Homenaje al Abate H. Breuil, t. II, pp. 135-139.

Rivero, O. (2009): "Les représentations de têtes de bison isolées du Magdalénien moyen franco-cantabrique : analyse des données technologiques et stylistiques", Préhistoire, art et sociétés, 64, pp. 175-184.

Ruiz-Redondo, A. (2014): Entre el Cantábrico y los Pirineos: el conjunto de Altxerri en el contexto de la actividad gráfica magdaleniense. Santander: Nadir.

Vialou, D. (1986): L'art des grottes en Ariège magdalénienne. Suppl. Gallia Préhistoire, xxII. Paris. 